\title{
Subcubic Equivalences Between Path, Matrix, and Triangle Problems*
}

\author{
Virginia Vassilevska Williams $^{\dagger} \quad$ Ryan Williams $^{\ddagger}$
}

\begin{abstract}
We say an algorithm on $n \times n$ matrices with entries in $[-M, M]$ (or $n$-node graphs with edge weights from $[-M, M])$ is truly subcubic if it runs in $O\left(n^{3-\delta} \cdot \operatorname{poly}(\log M)\right)$ time for some $\delta>0$. We define a notion of subcubic reducibility, and show that many important problems on graphs and matrices solvable in $O\left(n^{3}\right)$ time are equivalent under subcubic reductions. Namely, the following weighted problems either all have truly subcubic algorithms, or none of them do:

- The all-pairs shortest paths problem on weighted digraphs (APSP).

- Detecting if a weighted graph has a triangle of negative total edge weight.

- Listing up to $n^{2.99}$ negative triangles in an edge-weighted graph.

- Finding a minimum weight cycle in a graph of non-negative edge weights.

- The replacement paths problem on weighted digraphs.

- Finding the second shortest simple path between two nodes in a weighted digraph.

- Checking whether a given matrix defines a metric.

- Verifying the correctness of a matrix product over the $(\min ,+)$-semiring.

Therefore, if APSP cannot be solved in $n^{3-\varepsilon}$ time for any $\varepsilon>0$, then many other problems also need essentially cubic time. In fact we show generic equivalences between matrix products over a large class of algebraic structures used in optimization, verifying a matrix product over the same structure, and corresponding triangle detection problems over the structure. These equivalences simplify prior work on subcubic algorithms for all-pairs path problems, since it now suffices to give appropriate subcubic triangle detection algorithms.

Other consequences of our work are new combinatorial approaches to Boolean matrix multiplication over the (OR,AND)-semiring (abbreviated as BMM). We show that practical advances in triangle detection would imply practical BMM algorithms, among other results. Building on our techniques, we give two new BMM algorithms: a derandomization of the recent combinatorial BMM algorithm of Bansal and Williams (FOCS'09), and an improved quantum algorithm for BMM.
\end{abstract}

\footnotetext{
${ }^{*}$ This work originated while the authors were members of the Institute for Advanced Study, Princeton, NJ and visiting the Computer Science Department at Princeton University.

${ }^{\dagger}$ Computer Science Division, UC Berkeley, Berkeley, CA. Supported by a CRA Computing Innovations Fellowship. Email: virgi@eecs.berkeley.edu

${ }^{\ddagger}$ IBM Almaden Research Center, San Jose, CA. Supported by the Josef Raviv Memorial Fellowship. Email: ryanwilleus.ibm.com
} 


\section{Introduction}

Many computational problems on graphs and matrices have natural cubic time solutions. For example, $n \times n$ matrix multiplication over any algebraic structure can be done in $O\left(n^{3}\right)$ operations. For algebraic structures that arise in optimization, such as the $(\mathrm{min},+)$-semiring, it is of interest to determine when we need only a subcubic number of operations 11 The all-pairs shortest paths problem (APSP) also has a cubictime algorithm, known for over 40 years [Flo62, War62]. One of the "Holy Grails" of graph algorithms is to determine whether this cubic complexity is basically inherent, or whether a significant improvement (say, $O\left(n^{2.9}\right)$ time) is possible. (It is known that this question is equivalent to finding a faster algorithm for $(\min ,+)$ matrix multiplication. [FM71, Mun71]) Most researchers believe that cubic time is essentially necessary: there are $n^{2}$ pairs of nodes, and in the worst case we should not expect to improve too much on $\Omega(n)$ time per pair. (We should note that a long line of work has produced slightly subcubic algorithms with small poly $(\log n)$ improvements in the running time; the current best runs in $O\left(n^{3} \log \log ^{3} n / \log ^{2} n\right)$ time [Cha07].)

Related to APSP is the replacement paths problem (RPP): given nodes $s$ and $t$ in a weighted directed graph and a shortest path $P$ from $s$ to $t$, compute the length of the shortest simple path that avoids edge $e$, for all edges $e$ on $P$. This problem is studied extensively [Yen71, Law72, Epp98, KIM82, RZ05, Rod07, Ber10] for its applications to network reliability. A slightly subcubic time algorithm is not hard to obtain from a slightly subcubic APSP algorithm, but nothing faster than this is known. It does seem that cubic time may be inherent, since for all edges in a path (and there may be $\Omega(n)$ of them) we need to recompute a shortest path. A well-studied restriction of RPP is to find the second shortest (simple) path between two given nodes $s$ and $t$. Of course this problem also has a cubic algorithm, but again nothing much faster is known. However, the cubic complexity does not seem to be as vital: we simply want to find a certain type of path between two endpoints. Similarly, finding a minimum weight cycle in a graph with non-negative weights is only known to be possible in slightly subcubic time 2

An even simpler example is that of finding a triangle in an edge-weighted graph where the sum of edge weights is negative. Exhaustive search of all triples of nodes takes $O\left(n^{3}\right)$ time, and applying the best APSP algorithm makes this $O\left(n^{3} \log \log ^{3} n / \log ^{2} n\right)$ time, but we do not know a faster algorithm. Recent work has suggested that this negative triangle problem might have a faster algorithm, since the node-weighted version of the problem can be solved faster [VW06, VWY06, CL07]. (In fact the node-weighted version of the problem is no harder than the unweighted triangle detection problem, which is solvable in $O\left(n^{2.38}\right)$ time [IR78].) Since the cubic algorithm for negative triangle is so simple, and many restrictions of the problem have faster algorithms, it would appear that cubic complexity is unnecessary for finding a negative triangle.

We give theoretical evidence that these open algorithmic questions may be hard to resolve, by showing that they and other well-studied problems are all surprisingly equivalent, in the sense that there is a substantially subcubic algorithm for one of them if and only if all of them have substantially subcubic algorithms. Compare with the phenomenon of NP-completeness: one reason P vs NP looks so hard to resolve is that many researchers working in different areas have all been working on essentially the same (NP-complete) problem with no success. Our situation is entirely analogous: either these problems really need essentially cubic time, or we are missing a fundamental insight which would make all of them simultaneously easier.

\footnotetext{
${ }^{1}$ Note that in the specific case when the structure is a ring, it is well known that one can solve the problem much faster than $O\left(n^{3}\right)$ operations [Str69 CW90]. However it is unknown if this fact can be used to compute the matrix product fast on many other important structures such as commutative semirings.

${ }^{2}$ Note that if we allowed negative weights, this problem is immediately NP-hard.
} 
We say that an algorithm on $n \times n$ matrices (or an $n$-node graph) computing a set of values in $\{-M, \ldots, M\}$ is truly subcubic if it uses $O\left(n^{3-\delta} \cdot \operatorname{poly}(\log M)\right)$ time for some $\delta>0$. In general, poly $\log M$ factors are natural: the truly subcubic ring matrix multiplication algorithms have poly $\log M$ overhead if one counts the bit complexity of operations. In Appendix Fwe develop subcubic reductions between many problems, proving:

Theorem 1.1 The following problems either all have truly subcubic algorithms, or none of them do:

1. The all-pairs shortest paths problem on weighted digraphs (APSP).

2. The all-pairs shortest paths problem on undirected weighted graphs.

3. Detecting if a weighted graph has a triangle of negative total edge weight.

4. Listing up to $n^{2.99}$ negative triangles in an edge-weighted graph.

5. Verifying the correctness of a matrix product over the (min, + )-semiring.

6. Checking whether a given matrix defines a metric.

7. Finding a minimum weight cycle in a graph of non-negative edge weights.

8. The replacement paths problem on weighted digraphs.

9. Finding the second shortest simple path between two nodes in a weighted digraph.

Note the only previously known equivalence in the above was that of (1) and (2).

An explicit definition of our reducibility concept is given in Appendix $\mathrm{C}$ The truly subcubic runtimes may vary depending on the problem: given our reductions, an $\tilde{O}\left(n^{2.9}\right)$ algorithm for negative triangle implies an $\tilde{O}\left(n^{2.96}\right)$ algorithm for APSP. However, asymptotic runtime equivalences hold with respect to polylogarithmic improvements. That is, for each $c \geq 2$, the above either all have $O\left(\frac{n^{3}}{\log ^{c} n} \cdot\right.$ poly $\left.\log M\right)$ algorithms, or none of them do. Hence an $\Omega\left(n^{3} / \log ^{2} n\right)$ lower bound on APSP would imply a similar lower bound on all the above (within poly $\log M$ factors).

Perhaps the most interesting aspect of Theorem 1.1 is that some of the problems are decision problems and others are functions. Hence to prove lower bounds on some decision problems, it suffices to prove them on analogous multi-output functions. It is counterintuitive that an $O\left(n^{2.9}\right)$ algorithm returning one bit can be used to compute a function on $\Omega\left(n^{2}\right)$ bits in $O\left(n^{2.96}\right)$ time. Nevertheless, it is possible and in retrospect, our reductions are very natural. See Appendix $\mathrm{A}$ for some intuition.

A few equivalences in Theorem 1.1 follow from a more general theorem, which can be used to simplify prior work on all-pairs path problems. In general we consider (min, $\odot$ ) structures defined over a set $R \subset \mathbb{Z}$ together with an operation $\odot: R \times R \rightarrow \mathbb{Z} \cup\{-\infty, \infty\} 3$ We define a type of $(\mathrm{min}, \odot)$ structure that we call extended, which allows for an "identity matrix" and an "all-zeroes matrix" over the structure. (For definitions, see the Preliminaries.) Almost all structures we consider in this paper are extended, including the Boolean semiring over OR and AND, the (min, $\max )$-semiring, and the $(\min ,+)$-semiring. In Section 3 we prove:

Theorem 1.2 (Informal Statement of Theorems 3.1 and 3.2) Let $\overline{\mathcal{R}}$ be an extended $(\mathrm{min}, \odot)$ structure. The following problems over $\overline{\mathcal{R}}$ either all have truly subcubic algorithms, or none of them do:

- Negative Triangle Detection. Given an n-node graph with weight function $w: V \times V \rightarrow R \cup \mathbb{Z}$, find nodes $i, j, k$ such that $w(i, j) \in \mathbb{Z}, w(i, k) \in R, w(k, j) \in R$, and $(w(i, j) \odot w(k, j))+w(i, j)<0$.

\footnotetext{
${ }^{3} \mathrm{An}$ analogous treatment is possible for $(\max , \odot)$ structures. We omit the details, as they merely involve negations of entries.
} 
- Matrix Product. Given two $n \times n$ matrices $A$, $B$ with entries from $R$, compute the product of $A$ and $B$ over $\overline{\mathcal{R}}$.

- Matrix Product Verification. Given three $n \times n$ matrices $A, B, C$ with entries from $R$, determine if the product of $A$ and $B$ over $\overline{\mathcal{R}}$ is $C$.

The relationship between matrix product verification and the matrix product is particularly surprising, as $n \times n$ matrix product verification over rings can be done in $O\left(n^{2}\right)$ randomized time [BK95] but we do not know how to apply this fast verification to ring matrix multiplication. Spinrad [Spi03] (Open Problem 8.2) and Alon [Alo09] have asked if the verification problem for various matrix products can be done faster than the products themselves. Our reductions rely crucially on the fact that the addition operation in a $(\mathrm{min}, \odot)$ structure is a minimum.

In Appendix G, we show how our techniques can also be used to design alternative approaches to matrix multiplication over OR and AND. First we have as a consequence of Theorem 1.2;

Theorem 1.3 The following all have truly subcubic combinatorial algorithms, or none of them do:

- Boolean matrix multiplication (BMM).

- Detecting if a graph has a triangle.

- Listing up to $n^{2.99}$ triangles in a graph.

- Verifying the correctness of a matrix product over the Boolean semiring.

These reductions have low overhead, hence any simple fast triangle algorithm would yield a simple (and only slightly slower) BMM algorithm. This is a problem that has been investigated by many researchers, e.g. ([Woe08], Open Problem 4.3(c)) and ([Spi03], Open Problem 8.1). More concretely, Theorem 1.3]can already yield new BMM algorithms, with a little extra work. First, we can derandomize the recent combinatorial BMM algorithm of Bansal and Williams [BW09]:

Theorem 1.4 There is a deterministic combinatorial $O\left(n^{3} / \log ^{2.25} n\right)$-time algorithm for BMM.

The BMM algorithm of [BW09] uses randomness in two different ways: it reduces BMM to a graph theoretic problem, computes a pseudoregular partition of the graph in randomized quadratic time, then it uses random samples of nodes along with the partition to speed up the solution of the graph problem. We can avoid the random sampling by giving a triangle algorithm with $O\left(n^{3} / \log ^{2.25} n\right)$ running time, and applying Theorem 1.3, To get a deterministic triangle algorithm, we show (using a new reduction) that in fact any polynomial time algorithm for pseudoregularity suffices to get a subcubic triangle algorithm. With this relaxed condition, we can replace the randomized quadratic algorithm for pseudoregularity with a deterministic polynomial time algorithm of Alon and Naor [AN06].

We also obtain an improved quantum algorithm for BMM:

Theorem 1.5 There is an $\tilde{O}\left(\min \left\{n^{1.3} L^{17 / 30}, n^{2}+L^{47 / 60} n^{13 / 15}\right\}\right)$ quantum algorithm for computing the product of two $n \times n$ Boolean matrices, where $L$ is the number of ones in the output matrix.

Theorem 1.5 is obtained by simply applying the best known quantum algorithm for triangle [MSS05] to our reduction, improving the previous best [Bv06] output-sensitive quantum algorithm for BMM.

Remark. We have spent much time simplifying our proofs (contrary to all advice we have received), since one of our goals is to eventually uncover matrix multiplication algorithms that are more practical than what is 
currently known. Earlier versions of our reductions were extremely technical and gave very little insight; we hope our simplifications will be useful in future work.

\section{Preliminaries}

Unless otherwise noted, all graphs have $n$ vertices. Whenever an algorithm in our paper uses $\infty$ or $-\infty$, these can be substituted by numbers of suitably large absolute value. We use $\omega$ to denote the smallest real number such that $n \times n$ matrix multiplication over an arbitrary ring can be done in $O\left(n^{\omega}\right)$ operations.

Structures and Extended Structures. We give a general definition encompassing all algebraic structures for which our results apply. Let $R$ be a finite set. A (min, $\odot)$ structure over $R$ is defined by a binary operation $\odot: R \times R \rightarrow \mathbb{Z} \cup\{-\infty, \infty\}$. We use the variable $\mathcal{R}$ to refer to a (min, $\odot$ ) structure. We say a (min, $\odot$ ) structure is extended if $R \subset \mathbb{Z}$ and $R$ contains elements $\varepsilon_{0}$ and $\varepsilon_{1}$ such that for all $x \in R, x \odot \varepsilon_{0}=\varepsilon_{0} \odot x=\infty$ and $\varepsilon_{1} \odot x=x$ for all $x \in R$. That is, $\varepsilon_{0}$ is a type of annihilator, and $\varepsilon_{1}$ is a left identity. We use the variable $\overline{\mathcal{R}}$ to refer to an extended structure. The elements $\varepsilon_{0}$ and $\varepsilon_{1}$ allow us to define (for every $n$ ) an $n \times n$ identity matrix $I_{n}$ and a $n \times n$ zero matrix $Z_{n}$ over $\overline{\mathcal{R}}$. More precisely, $I_{n}[i, j]=\varepsilon_{0}$ for all $i \neq j, I_{n}[i, i]=\varepsilon_{1}$, and $Z_{n}[i, j]=\varepsilon_{0}$ for all $i, j$. We shall omit the subscripts of $I_{n}$ and $Z_{n}$ when the dimension is clear.

Examples of extended structures $\overline{\mathcal{R}}$ are the $(O R, A N D)$ (or Boolean) semiring 4 as well as the (min, $\max$ ) and (min, + ) semirings (also called subtropical and tropical), and the $(\mathrm{min}, \leq)$ structure used to solve all pairs earliest arrivals [Vas08]. An example of a structure that is not extended is the "existence dominance" structure defined in Appendix $\mathrm{H}$.

Matrix Products Over Structures. The matrix product of two $n \times n$ matrices over $\mathcal{R}$ is

$$
(A \odot B)[i, j]=\min _{k \in[n]}(A[i, k] \odot B[k, j]) .
$$

It is easy to verify that for all matrices $A$ over an extended $\overline{\mathcal{R}}, I \odot A=A$ and $Z \odot A=A \odot Z=F$ where $F[i, j]=\infty$ for all $i, j$. The problem of matrix product verification over an extended structure $\overline{\mathcal{R}}$ is to determine whether $\min _{k \in[n]}(A[i, k] \odot B[k, j])=C[i, j]$ for all $i, j \in[n]$, where $A, B, C$ are given $n \times n$ matrices with entries from $R$. Although it looks like a simpler problem, matrix product verification for the $(\mathrm{min},+)$ semiring (for instance) is not known to have a truly subcubic algorithm.

Negative Triangles Over Structures. The negative triangle problem over $\mathcal{R}$ is defined on a weighted tripartite graph with parts $I, J, K$. Edge weights between $I$ and $J$ are from $\mathbb{Z}$, and all other edge weights are from $R$. The problem is to detect if there are $i \in I, j \in J, k \in K$ so that $(w(i, k) \odot w(k, j))+w(i, j)<0$. Note that if one negates all weights of edges between $I$ and $J$, the condition becomes $(w(i, k) \odot w(k, j))<w(i, j)$. In the special case when $\odot=+$ and $R \subseteq \mathbb{Z} \cup\{-\infty, \infty\}$, the tripartiteness requirement is unnecessary, and the negative triangle problem is defined on an arbitrary graph with edge weights from $\mathbb{Z} \cup\{-\infty, \infty\}$. This holds for the negative triangle problem over both the $(\min ,+)$ and Boolean semirings.

\subsection{Prior Work}

Since our paper covers many popular problems in the literature, there is much relevant prior work. Due to lack of space, we highlight the most relevant sources in Appendix B,

\footnotetext{
${ }^{4}$ Observe the Boolean semiring is isomorphic to the structure on elements $\varepsilon_{0}=\infty$ and $\varepsilon_{1}=0$, where $x \odot y=x+y$.
} 


\section{Equivalences Between Problems on Generic Structures}

A generic approach to computing fast $(\min , \odot)$ matrix products (for an arbitrary binary operation $\odot$ ) would be of major interest. Here we prove truly subcubic equivalences between matrix products, negative triangles, and matrix product verification for $(\mathrm{min}, \odot)$ structures. (For definitions, see the Preliminaries.)

Reminder of Theorems 3.1 and 3.2 Let $\overline{\mathcal{R}}$ be an extended $(\mathrm{min},+)$ structure. The following problems over $\overline{\mathcal{R}}$ either all have truly subcubic algorithms, or none of them do:

- Negative Triangle Detection. Given an n-node graph with weight function $w: V \times V \rightarrow R \cup \mathbb{Z}$, find nodes $i, j, k$ such that $w(i, j) \in \mathbb{Z}, w(i, k) \in R, w(k, j) \in R$, and $(w(i, j) \odot w(k, j))+w(i, j)<0$.

- Matrix Product. Given two $n \times n$ matrices $A, B$ with entries from $R$, compute the product of $A$ and $B$ over $\overline{\mathcal{R}}$.

- Matrix Product Verification. Given three $n \times n$ matrices $A, B, C$ with entries from $R$, determine if the product of $A$ and $B$ over $\overline{\mathcal{R}}$ is $C$.

\subsection{Negative Triangle Detection Implies Matrix Product Verification}

We start by showing that matrix product verification can solve the negative triangle problem over any extended structure $\overline{\mathcal{R}}$ in the same asymptotic runtime. For two problems $A$ and $B$, we write $A \leq_{3} B$ to express that there is a subcubic reduction from $A$ to $B$. (For formal definitions, see Appendix $[$ )

Theorem 3.1 (Negative Triangle Over $\overline{\mathcal{R}} \leq_{3}$ Matrix Product Verification Over $\overline{\mathcal{R}}$ ) Suppose matrix product verification over $\overline{\mathcal{R}}$ can be done in time $T(n)$. Then the negative triangle problem for graphs over $\overline{\mathcal{R}}$ can be solved in $O(T(n))$ time.

Proof. From the tripartite graph $G=(I \cup J \cup K, E)$ given by the negative triangle problem over $\overline{\mathcal{R}}$, construct matrices $A, B, C$ as follows. For each edge $(i, j) \in(I \times J) \cap E$ set $C[i, j]=w(i, j)$. Similarly, for each edge $(i, k) \in(I \times K) \cap E$ set $A[i, k]=w(i, k)$ and for each edge $(k, j) \in(K \times J) \cap E$ set $B[k, j]=w(k, j)$. When there is no edge in the graph, the corresponding matrix entry in $A$ or $B$ becomes $\varepsilon_{0}$ and in $C$ it becomes $\infty$. The problem becomes to determine whether there are $i, j, k \in[n]$ so that $A[i, k] \odot$ $B[k, j]<C[i, j]$. Let $A^{\prime}$ be the $n \times 2 n$ matrix obtained by concatenating $A$ to the left of the $n \times n$ identity matrix $I$. Let $B^{\prime}$ be the $2 n \times n$ matrix obtained by concatenating $B$ on top of $C$. Then $A^{\prime} \odot B^{\prime}$ is equal to the componentwise minimum of $A \odot B$ and $C$. One can complete $A^{\prime}, B^{\prime}$ and $C$ to square $2 n \times 2 n$ matrices by concatenating an all $\varepsilon_{0} n \times 2 n$ matrix to the bottom of $A^{\prime}$, an all $\varepsilon_{0} 2 n \times n$ matrix to the right of $B^{\prime}$ and $n$ columns of all $\varepsilon_{0} \mathrm{~s}$ and $n$ rows of all $\varepsilon_{0}$ s to the right and bottom of $C$ respectively.

Run matrix product verification on $A^{\prime}, B^{\prime}, C$. Suppose there are some $i, j$ so that $\min _{k}\left(A^{\prime}[i, k] \odot B^{\prime}[k, j]\right) \neq$ $C[i, j]$. Then since

$$
\min _{k}\left(A^{\prime}[i, k] \odot B^{\prime}[k, j]\right)=\min \left\{C[i, j], \min _{k}(A[i, k] \odot B[k, j])\right\} \leq C[i, j],
$$

there must exists a $k \in[n]$ so that $A[i, k] \odot B[k, j]<C[i, j]$. In other words, $i, k, j$ is a negative triangle over $\overline{\mathcal{R}}$. If on the other hand for all $i, j$ we have $\min _{k}\left(A^{\prime}[i, k] \odot B^{\prime}[k, j]\right)=C[i, j]$, then for all $i, j$ we have $\min _{k}(A[i, k] \odot B[k, j]) \geq C[i, j]$ and there is no negative triangle. 


\subsection{Negative Triangle Detection Implies Matrix Multiplication}

Next we show that from negative triangle detection over a $(\min , \odot)$ structure $\mathcal{R}$, we can obtain the full matrix product over $\mathcal{R}$. Specifically, we prove the following.

Theorem 3.2 (Matrix Product Over $\mathcal{R} \leq_{3}$ Negative Triangle Over $\mathcal{R}$ ) Let $T(n)$ be a function so that $T(n) / n$ is nondecreasing. Suppose the negative triangle problem over $\mathcal{R}$ in an n-node graph can be solved in $T(n)$ time. Then the product of two $n \times n$ matrices over $\mathcal{R}$ can be performed in $O\left(n^{2} \cdot T\left(n^{1 / 3}\right) \log W\right)$ time, where $W$ is the absolute value of the largest finite integer in the output.

Before we proceed, let us state some simple but useful relationships between triangle detecting, finding, and listing. The proofs of the Lemma 3.1 and Theorem 3.3 appear in Appendix D.

Lemma 3.1 (Folklore) Let $T(n)$ be a function so that $T(n) / n$ is nondecreasing. If there is a $T(n)$ time algorithm for negative triangle detection over $\mathcal{R}$ on a graph $G=(I \cup J \cup K, E)$, then there is an $O(T(n))$ algorithm which returns a negative triangle over $\mathcal{R}$ in $G$ if one exists.

It will be useful in our final algorithm to have a method for finding many triangles, given an algorithm that can detect one. We can extend Lemma 3.1 in a new way, to show that subcubic negative triangle detection implies subcubic negative triangle listing, provided that the number of negative triangles to be listed is subcubic.

Theorem 3.3 (Negative Triangle Listing Over $\mathcal{R} \leq_{3}$ Negative Triangle Over $\mathcal{R}$ ) Suppose there is a truly subcubic algorithm for negative triangle detection over $\mathcal{R}$. Then there is a truly subcubic algorithm which lists $\Delta$ negative triangles over $\mathcal{R}$ in any graph with at least $\Delta$ negative triangles, for any $\Delta=O\left(n^{3-\delta}\right)$, $\delta>0$.

Next we show that fast negative triangle detection over $\mathcal{R}$ implies a fast algorithm for finding many edgedisjoint negative triangles over $\mathcal{R}$. Consider a tripartite graph with parts $I, J, K$. We say a set of triangles $T \subseteq I \times J \times K$ in the graph is $I J$-disjoint if for all $(i, j, k) \in T,\left(i^{\prime}, j^{\prime}, k^{\prime}\right) \in T,(i, j) \neq\left(i^{\prime}, j^{\prime}\right)$.

Lemma 3.2 Let $T(n)$ be a function so that $T(n) / n$ is nondecreasing. Given a $T(n)$ algorithm for negative triangle detection over $\mathcal{R}$, there is an algorithm $A$ which outputs a maximal set $L$ of IJ-disjoint negative triangles over $\mathcal{R}$ in a tripartite graph with distinguished parts $(I, J, K)$, in $O\left(T\left(n^{1 / 3}\right) n^{2}\right)$ time. Furthermore, if there is a constant $\varepsilon: 0<\varepsilon<1$ such that for all large enough $n, T(n) \geq T\left(2^{1 / 3} n\right) /(2(1-\varepsilon))$, then there is an output-sensitive $O\left(T\left(n /|L|^{1 / 3}\right)|L|\right)$-time algorithm.5

In particular, Lemma 3.2 implies that given any graph on $n$ nodes, we can determine those pairs of nodes that lie on a negative triangle in $O\left(T\left(n^{1 / 3}\right) n^{2}\right)$ time. The condition required for the output sensitive algorithm holds for all subcubic polynomials, but it does not necessarily hold for runtimes of the form $n^{3} / f(n)$ with $f(n)=n^{o(1)}$. In the special case when $T(n)$ is $\Theta\left(n^{3} / \log ^{c} n\right)$ for a constant $c$, the output sensitive algorithm only multiplies a $\log |L|$ factor to the runtime.

Proof. Algorithm $A$ maintains a global list $L$ of negative triangles over $\mathcal{R}$ which is originally empty and will be the eventual output of the algorithm. Let $a$ be a parameter to be set later. At each point the algorithm works with a subgraph $\tilde{G}$ of the original graph, containing all of the nodes, all of the edges between $I$ and $K$ and between $J$ and $K$ but only a subset of the edges between $I$ and $J$. In the beginning $\tilde{G}=G$ and at each step $A$ removes an edge from $\tilde{G}$.

\footnotetext{
${ }^{5}$ The condition is satisfied for instance when $T(n) / n^{3-\delta}$ is nonincreasing for some $\delta>0$.
} 
Algorithm $A$ starts by partitioning each set $I, J, K$ into $n^{a}$ parts where each part has at most $\left\lceil n^{(1-a)}\right\rceil$ nodes each. It iterates through all $n^{3 a}$ possible ways to choose a triple of parts $\left(I^{\prime}, J^{\prime}, K^{\prime}\right)$ so that $I^{\prime} \subset I$, $J^{\prime} \subset J$ and $K^{\prime} \subset K$. For each triple $\left(I^{\prime}, J^{\prime}, K^{\prime}\right)$ in turn, it considers the subgraph $G^{\prime}$ of $\tilde{G}$ induced by $I^{\prime} \cup J^{\prime} \cup K^{\prime}$ and repeatedly uses Lemma 3.1 to return a negative triangle over $\mathcal{R}$. Each time a negative triangle $(i, j, k)$ is found in $G^{\prime}$, the algorithm adds $(i, j, k)$ to $L$, removes edge $(i, j)$ from $\tilde{G}$ and attempts to find a new negative triangle in $G^{\prime}$. This process repeats until $G^{\prime}$ contains no negative triangles, in which case algorithm $A$ moves on to the next triple of parts.

Now, let us analyze the running time of $A$. For a triple of parts $\left(I^{\prime}, J^{\prime}, K^{\prime}\right)$ let $e_{I^{\prime} J^{\prime} K^{\prime}}$ be the number of edges $(i, j)$ in $I^{\prime} \times J^{\prime}$ that are found in the set of $I^{\prime} J^{\prime}$-disjoint negative triangles when $\left(I^{\prime}, J^{\prime}, K^{\prime}\right)$ is processed by $A$. Let $T(n)$ be the complexity of negative triangle detection over $\mathcal{R}$. Then the runtime can be bounded from above as:

$$
O\left(\sum_{\text {all } n^{3 a} \text { triples } I^{\prime}, J^{\prime}, K^{\prime}}\left(e_{I^{\prime} J^{\prime} K^{\prime}} \cdot T\left(n^{1-a}\right)+T\left(n^{1-a}\right)\right)\right) .
$$

Note that the sum of all $e_{I^{\prime} J^{\prime} K^{\prime}}$ is at most $n^{2}$, since if edge $(i, j) \in I^{\prime} \times J^{\prime}$ is reported to be in a negative triangle, then it is removed from the graph. Hence there is a constant $c>0$ such that (1) is upper bounded by:

$$
\begin{aligned}
c \cdot T\left(n^{1-a}\right) \cdot \sum_{\text {all } n^{3 a} \text { triples } I^{\prime}, J^{\prime}, K^{\prime}}\left(e_{I^{\prime} J^{\prime} K^{\prime}}+1\right) & \leq c \cdot T\left(n^{1-a}\right) \cdot\left(n^{3 a}+\sum_{\text {all } n^{3 a}} e_{\text {triples } I^{\prime}, J^{\prime}, K^{\prime}} e_{I^{\prime} J^{\prime} K^{\prime}}\right) \\
& \leq c \cdot T\left(n^{1-a}\right) \cdot\left(n^{3 a}+n^{2}\right) .
\end{aligned}
$$

Setting $a=2 / 3$, the runtime becomes $O\left(n^{2} T\left(n^{1 / 3}\right)\right)$.

To get an output-sensitive algorithm $A^{\prime}$, we make the following modification. For all $i=1, \ldots, 2 \log n$, run algorithm $A$ with $a:=i /(3 \log n)$, and stop when the list $L$ contains at least $2^{i}$ edges. If $|L|=\left|L_{i-1}\right|$ then return $L$; otherwise set $L_{i}:=L$ and continue with stage $i+1$.

The runtime of $A^{\prime}$ is

$$
\begin{aligned}
& \sum_{i=1}^{\log |L|} T\left(n^{1-i /(3 \log n)}\right) \cdot\left(n^{3 i /(3 \log n)}+\sum_{\text {all } n^{3 i /(3 \log n) \text { triples } I^{\prime}, J^{\prime}, K^{\prime}}}\left(e_{I^{\prime} J^{\prime} K^{\prime}}\right)\right) \leq \\
& \sum_{i=1}^{\log |L|}\left(n^{i / \log n}+2^{i}\right) \cdot T\left(n^{1-i /(3 \log n)}\right)=2 \sum_{i=1}^{\log |L|} 2^{i} T\left(2^{\log n-i / 3}\right)=2 \sum_{i=1}^{\log |L|} 2^{i} T\left(n / 2^{i / 3}\right) .
\end{aligned}
$$

Since there is a constant $\varepsilon<1$ so that for all $n, T(n) \geq T\left(2^{1 / 3} n\right) /(2(1-\varepsilon))$, then for all $i, 2^{i} T\left(n / 2^{i / 3}\right) \leq$ $2^{i+1}(1-\varepsilon) T\left(n / 2^{(i+1) / 3}\right)$ and hence the runtime is bounded by

$$
O\left(T\left(n /|L|^{1 / 3}\right)|L| \sum_{i=0}^{\log |L|}(1-\varepsilon)^{i}\right)=O\left(T\left(n /|L|^{1 / 3}\right)|L|\right)
$$


We are now ready to prove Theorem 3.2 , via a simultaneous binary search on entries of the matrix product. The "oracle" used for binary search is our algorithm for $I J$-disjoint triangles.

Proof of Theorem 3.2. Let $A$ and $B$ be the given $n \times n$ matrices. Suppose the integers in the output $A \odot B$ lie in $[-W, W] \cup\{\infty,-\infty\}$. We will binary search on $[-W, W]$ for the finite entries.

We maintain two $n \times n$ matrices $S$ and $H$ so that originally $S[i, j]=-W$ and $H[i, j]=W+1$ for all $i, j \in[n]$. The algorithm proceeds in iterations. In each iteration a complete tripartite graph $G$ is created on partitions $I, J$ and $K$. The edges of $G$ have weights $w(\cdot)$ so that for $i \in I, j \in J$ and $k \in K, w(i, k)=A[i, k]$, $w(k, j)=B[k, j]$ and $w(i, j)=\lceil(S[i, j]+H[i, j]) / 2\rceil$. After this, using the algorithm from Lemma 3.2. generate a list $L$ of $I J$-disjoint negative triangles over $\mathcal{R}$ for $G$ in $O(T(n))$ time. Now, modify $S$ and $H$ as follows. If $(i, j)$ appears in a triangle in $L$ for $i \in I, j \in J$, then $H[i, j]=w(i, j)$, otherwise $S[i, j]=w(i, j)$. Continue iterating until for all $i, j, H[i, j] \leq S[i, j]+1$.

Finally, create the result matrix $C$. To compute the entries of $C$, set up a complete tripartite graph $G$ on partitions $I, J$ and $K$. The edges of $G$ have weights $w(\cdot)$ so that for $i \in I, j \in J$ and $k \in K, w(i, k)=A[i, k]$, $w(k, j)=B[k, j]$ and $w(i, j)=S[i, j]$. Use the algorithm from Lemma 3.2 to obtain a list $L$ of $I J$ disjoint negative triangles in $O(T(n))$ time. For all $i \in I, j \in J$ so that $(i, j)$ appears in a triangle in $L$, set $C[i, j]=S[i, j]$; otherwise, set $C[i, j]=H[i, j]$.

Corollary 3.1 Suppose the negative triangle problem over $\mathcal{R}$ is in $O\left(n^{3} / \log ^{c} n\right)$ time for some constant c. Then the product of $n \times n$ matrices over $\mathcal{R}$ can be done in $O\left((\log W) n^{3} / \log ^{c} n\right)$ time.

An important special case of matrix multiplication is that of multiplying rectangular matrices. Negative triangle detection can also give a speedup in this case as well.

Theorem 3.4 Suppose the negative triangle problem over $\mathcal{R}$ is in $T(n)$ time. Then two matrices of dimensions $m \times n$ and $n \times p$ can be multiplied over $\mathcal{R}$ in $O\left(m p \cdot T\left(n^{1 / 3}\right) \log W\right)$ time, where the entries in the output lie in $[-W, W] \cup\{-\infty, \infty\}$.

If $T(n)=n^{c}$ the runtime is $O\left(m p(n)^{c / 3}\right)$. Notice that if $c<3$ and if $p=n^{(3-c) / 3}$, then the runtime would be $O(m n)$. That is, for any $c<3$, there is some $p \geq n^{\varepsilon}$ such that multiplication of $m \times n$ and $n \times p$ matrices over $\mathcal{R}$ can be done optimally. Similar to Lemma 3.2, for most functions $T(n)$, the result can be modified to give an output-sensitive $O\left(\ell \cdot T\left((m n p / \ell)^{1 / 3}\right)\right)$-time algorithm for $m \times n$ and $n \times p$ matrix product over $\mathcal{R}$, where $\ell$ is the number of ones in the product matrix. The proof of Theorem 3.4 appears in Appendix $\mathrm{E}$

\section{Discussion}

In this conference submission, we can only include a few of our results within the first ten pages. The remaining results appear in appendices. (The full appendix-free version will be available on the authors' webpages.) Here we summarize the results contained in these appendices for convenience.

\subsection{Problems Equivalent to All-Pairs Shortest Paths.}

In Appendix F we prove Theorem 1.1, which is a series of subcubic equivalences between the All-Pairs Shortest Paths (APSP) problem, Negative Edge-Weight Triangle, and other related problems. Most of the equivalences follow from Theorem 1.2 in the special case where the structure $\overline{\mathcal{R}}$ is the $(\mathrm{min},+)$-semiring, or by reweighting tricks. However, the equivalences concerning the Replacement Paths and Second Shortest Simple Path problems require new reductions. We show that they are equivalent to the others by showing that 
they can be used to detect a negative triangle. (It is known that they can be reduced to APSP.)

\subsection{Boolean Matrix Multiplication and Related Problems.}

In Appendix G, we show how our techniques can be used to provide alternative algorithms for BMM. It follows from Theorem 1.2 that triangle detection in an unweighted graph, Boolean matrix multiplication, and verifying the Boolean product of two matrices have fast and practical reductions between each other, so that any fast practical algorithm for one would entail similar algorithms for the other two.

Roditty and Zwick [RZ05] give a combinatorial algorithm for the second shortest simple path problem in unweighted directed graphs that runs in $O(m \sqrt{n} \log n)$. We show that a polylog improvement on their algorithm would imply a new subcubic algorithm for BMM.

Theorem 4.1 Suppose there exist nondecreasing functions $f(n)$ and $m(n)$ with $m(n) \geq n$, and a combinatorial algorithm which runs in $O(m(n) \sqrt{n} / f(n))$ time and computes the second shortest simple path in any given unweighted directed graph with $n$ nodes and $m(n)$ edges. Then there is a combinatorial algorithm for triangle detection running in $O\left(n^{3} / f(n)\right)$ time. If $f(n)=n^{\varepsilon}$ for some $\varepsilon>0$, then there is a truly subcubic combinatorial algorithm for BMM.

We also give two new BMM algorithms. First, we can derandomize Bansal and Williams' recent combinatorial BMM algorithm [BW09], which was the first to asympotically improve on the old Four Russians algorithm [ADKF70]. One step is to show that for the problem of preprocessing a graph to answer independent set queries fast, any polynomial time processing suffices to get faster BMM:

Theorem 4.2 Suppose there are $k, c>0$ such that every n-node graph can be preprocessed in $O\left(n^{k}\right)$ time so that all subsequent batches of $O(\log n)$ independent set queries $S_{1}, \ldots, S_{\log n}$ can be answered in $O\left(n^{2} / \log ^{c} n\right)$ time. Then triangle detection (and hence Boolean matrix multiplication) is solvable in $O\left(n^{3} / \log ^{c+1} n\right)$ time.

Generalizing Theorem 4.2, we can identify a natural query problem on weighted graphs whose solution would give faster APSP algorithms. On a graph with an edge weight function $c: E \rightarrow \mathbb{Z}$, define a price query to be an assignment of node weights $p: V \rightarrow \mathbb{Z}$, where a query answer is yes if and only if there is an edge $(u, v) \in E$ such that $p(u)+p(v)>c(u, v)$. Intuitively, think of $p(v)$ as a price on node $v$, the edge weight $c(u, v)$ as the cost of producing both $u$ and $v$, and we wish to find for a given list of prices if there is any edge we are willing to "sell" at those prices.

Theorem 4.3 Suppose there are $k, c>0$ such that every n-node edge-weighted graph can be preprocessed in $O\left(n^{k}\right)$ time so that any price query can be answered in $O\left(n^{2} / \log ^{c} n\right)$ time. Then negative triangle detection is solvable in $O\left(n^{3} / \log ^{c} n\right)$ time (and hence APSP is solvable in $O\left(n^{3} \log W / \log ^{c} n\right)$ time.

The contrapositive of Theorem 4.3 is interesting: assuming that APSP needs $\Omega\left(n^{3} / \operatorname{poly} \log n\right)$ time, there is a super-polynomial time lower bound on the preprocessing needed for efficiently answering price queries.

Our second BMM algorithm is a faster quantum BMM algorithm, obtained by a reduction to quantum triangle detection.

\subsection{A Simplified View of All-Pairs Path Problems and Their Matrix Products.}

In Appendix $\mathrm{H}$ we show how our equivalences can be used to simplify the constructions of subcubic algorithms for several special matrix products and all-pairs path problems in the literature: the existencedominance product, node-weighted APSP, all-pairs nondecreasing paths, and all-pairs bottleneck paths. The 
first two reduce to a special triangle detection problem, and the last two reduce to another type of triangle detection. We show that both triangle problems have simple subcubic algorithms.

\subsection{Extension to 3SUM.}

Using the ideas of the paper, in Appendix \ we show a subquadratic equivalence between the 3SUM problem and All-Ints 3SUM. In the 3SUM problem, one is given three lists $A, B, C$ of integers, and the goal is to determine if there are $a \in A, b \in B, c \in C$ such that $a+b+c=0$. An $O\left(n^{2}\right)$ algorithm is well-known and it is a celebrated open problem in computational geometry to find a much faster algorithm. The All-Ints 3SUM problem is a function version of the 3SUM problem: given the same lists $A, B, C$, now the goal is to determine all integers $a \in A$ such that there exist $b \in B, c \in C$ with $a+b+c=0$. Although this function version looks much harder, we prove that an $O\left(n^{2-\varepsilon}\right)$ algorithm for 3SUM implies an $O\left(n^{2-\varepsilon^{\prime}}\right)$ algorithm for All-Ints 3SUM. This may be seen as further evidence that the 3SUM problem is hard to solve substantially faster than quadratic time.

\section{Conclusion}

We have explored a new notion of reducibility which preserves truly subcubic runtimes. Our main contributions are subcubic reductions from important function problems (such as all-pairs paths and matrix products) to important decision problems (such as triangle detection and product verification), showing that subcubic algorithms for the latter entail subcubic algorithms for the former. We have shown that these reductions and the ideas behind them have many interesting consequences.

We conclude with three open questions arising from this work:

1. Does $O\left(n^{3-\delta}\right)$ negative triangle detection imply $O\left(n^{3-\delta}\right)$ matrix product (over any $\mathcal{R}$ )? Note we can currently show that $O\left(n^{3-\delta}\right)$ negative triangle implies $O\left(n^{3-\delta / 3}\right)$ matrix product.

2. Does a truly subquadratic algorithm for $3 S U M$ imply truly subcubic APSP? We believe truly subquadratic 3SUM should imply truly subcubic negative triangle, which would answer the question.

3. Is there a truly subcubic algorithm for minimum edge-weight triangle? Although it has been asked in prior work, clearly this question takes on a much stronger importance, now that we know it is equivalent to asking for a truly subcubic APSP algorithm.

\section{References}

[ADKF70] V. L. Arlazarov, E. A. Dinic, M. A. Kronrod, and I. A. Faradzev. On economical construction of the transitive closure of an oriented graph. Soviet Math. Dokl., 11:1209-1210, 1970.

[AGM97] N. Alon, Z. Galil, and O. Margalit. On the exponent of the all pairs shortest path problem. $J$. Comput. Syst. Sci., 54(2):255-262, 1997.

[Alo09] N. Alon. Personal communication. 2009.

[AN06] N. Alon and A. Naor. Approximating the cut-norm via Grothendieck's inequality. SIAM J. Computing, 35:787-803, 2006.

[BDP08] I. Baran, E.D. Demaine, and M. Patrascu. Subquadratic algorithms for 3sum. Algorithmica, 50(4):584-596, 2008. 
[BDST08] J. Brickell, I.S. Dhillon, S. Sra, and J.A. Tropp. The metric nearness problem. SIAM J. Matrix Anal. Appl., 30(1):375-396, 2008.

[Ber10] A. Bernstein. A nearly optimal algorithm for approximating replacement paths and $k$ shortest simple paths in general graphs. In Proc. SODA, volume 21, pages 742-755, 2010.

[BK95] M. Blum and S. Kannan. Designing programs that check their work. J. ACM, 42(1):269-291, 1995.

[Bv06] H. Buhrman and R. Špalek. Quantum verification of matrix products. In SODA '06: Proceedings of the seventeenth annual ACM-SIAM symposium on Discrete algorithm, pages 880-889, 2006.

[BW09] N. Bansal and R. Williams. Regularity lemmas and combinatorial algorithms. In Proc. FOCS, pages $745-754,2009$.

[Cha05] T. M. Chan. All-pairs shortest paths with real weights in $O\left(n^{3} / \log n\right)$ time. In Proc. WADS, volume 3608, pages 318-324, 2005.

[Cha07] T. M. Chan. More algorithms for all-pairs shortest paths in weighted graphs. In Proc. STOC, pages 590-598, 2007.

[CL07] A. Czumaj and A. Lingas. Finding a heaviest triangle is not harder than matrix multiplication. In Proc. SODA, pages 986-994, 2007.

[CW90] D. Coppersmith and S. Winograd. Matrix multiplication via arithmetic progressions. J. Symbolic Computation, 9(3):251-280, 1990.

[Die96] M. Dietzfelbinger. Universal hashing and $k$-wise independent random variables via integer arithmetic without primes. In Proc. STACS, pages 569-580, 1996.

[DP80] D. Dubois and H. Prade. Fuzzy sets and systems: Theory and applications. Academic Press, 1980.

[DP09] R. Duan and S. Pettie. Fast algorithms for (max, min)-matrix multiplication and bottleneck shortest paths. In SODA '09: Proceedings of the Nineteenth Annual ACM -SIAM Symposium on Discrete Algorithms, pages 384-391, 2009.

[Epp98] D. Eppstein. Finding the $k$ shortest paths. SIAM Journal on Computing, 28(2):652-673, 1998.

[FK99] A. M. Frieze and R. Kannan. Quick approximation to matrices and applications. Combinatorica, 19(2):175-220, 1999.

[Flo62] R. W. Floyd. Algorithm 97: shortest path. Comm. ACM, 5:345, 1962.

[FM71] M. J. Fischer and A. R. Meyer. Boolean matrix multiplication and transitive closure. In Proc. FOCS, pages 129-131, 1971.

[FT87] M. L. Fredman and R. E. Tarjan. Fibonacci heaps and their uses in improved network optimization algorithms. JACM, 34(3):596-615, 1987.

[GO95] A. Gajentaan and M. Overmars. On a class of $o\left(n^{2}\right)$ problems in computational geometry. Computational Geometry, 5(3):165-185, 1995. 
[HSB07] J. Hershberger, S. Suri, and A. Bhosle. On the difficulty of some shortest path problems. ACM TALG, 3(1):5, 2007.

[IR78] A. Itai and M. Rodeh. Finding a minimum circuit in a graph. SIAM J. Computing, 7(4):413-423, 1978.

[KIM82] N. Katoh, T. Ibaraki, and H. Mine. An efficient algorithm for $K$ shortest simple paths. Networks, 12(4):411-427, 1982.

[KKP93] D. Karger, D. Koller, and S. Phillips. Finding the hidden path: Time bounds for all-pairs shortest paths. SIAM J. Computing, 22(6):1199-1217, 1993.

[Law72] E.L. Lawler. A procedure for computing the $K$ best solutions to discrete optimization problems and its application to the shortest path problem. Management Science, 18:401-405, 1971/72.

[Lin09] A. Lingas. A fast output-sensitive algorithm for boolean matrix multiplication. In Proc. ESA, pages 408-419, 2009.

[Mat91] J. Matousek. Computing dominances in $E^{n}$. Information Processing Letters, 38(5):277-278, 1991.

[MSS05] F. Magniez, M. Santha, and M. Szegedy. Quantum algorithms for the triangle problem. In Proc. SODA, pages 1109-1117, Philadelphia, PA, USA, 2005. Society for Industrial and Applied Mathematics.

[Mun71] J. I. Munro. Efficient determination of the transitive closure of a directed graph. Inf. Process. Lett., 1(2):56-58, 1971.

[Pan78] V. Y. Pan. Strassen's algorithm is not optimal; trilinear technique of aggregating, uniting and canceling for constructing fast algorithms for matrix operations. In Proc. FOCS, volume 19, pages 166-176, 1978.

[Pan80] V. Y. Pan. New fast algorithms for matrix operations. SIAM J. Comput., 9(2):321-342, 1980.

[Rod07] L. Roditty. On the $K$-simple shortest paths problem in weighted directed graphs. In Proc. SODA, volume 18, pages 920-928, 2007.

[RZ04] L. Roditty and U. Zwick. On dynamic shortest paths problems. In ESA, pages 580-591, 2004.

[RZ05] L. Roditty and U. Zwick. Replacement paths and $k$ simple shortest paths in unweighted directed graphs. In Proc. ICALP, volume 32, pages 249-260, 2005.

[Spi03] J. P. Spinrad. Efficient graph representations. Fields Institute Monographs, 19, 2003.

[Str69] V. Strassen. Gaussian elimination is not optimal. Numer. Math., 13:354-356, 1969.

[SZ99] A. Shoshan and U. Zwick. All pairs shortest paths in undirected graphs with integer weights. In Proc. FOCS, pages 605-614, 1999.

[Vas08] V. Vassilevska. Nondecreasing paths in weighted graphs, or: how to optimally read a train schedule. In Proc. SODA, pages 465-472, 2008. 
[VW06] V. Vassilevska and R. Williams. Finding a maximum weight triangle in $n^{3-\delta}$ time, with applications. In Proc. STOC, pages 225-231, 2006.

[VWY06] V. Vassilevska, R. Williams, and R. Yuster. Finding the smallest $H$-subgraph in real weighted graphs and related problems. In Proc. ICALP, volume 4051, pages 262-273, 2006.

[VWY07] V. Vassilevska, R. Williams, and R. Yuster. All-pairs bottleneck paths for general graphs in truly sub-cubic time. In Proc. STOC, pages 585-589, 2007.

[War62] S. Warshall. A theorem on boolean matrices. J. ACM, 9(1):11-12, 1962.

[Wil07] R. Williams. Matrix-vector multiplication in sub-quadratic time (some preprocessing required). In Proc. SODA, pages 995-1001, 2007.

[Woe08] G. J. Woeginger. Open problems around exact algorithms. Discrete Applied Mathematics, 156(3):397 - 405, 2008. Combinatorial Optimization 2004, CO2004.

[Yen71] J. Y. Yen. Finding the $K$ shortest loopless paths in a network. Management Science, 17:712-716, 1970/71.

[Yuv76] G. Yuval. An algorithm for finding all shortest paths using $N^{2.81}$ infinite-precision multiplications. Inf. Proc. Letters, 4:155-156, 1976.

\section{A A Little Intuition}

One of our key observations is the counterintuitive result that subcubic algorithms for certain triangle detection problems can be used to obtain subcubic matrix products in many forms, including products that are not known to be subcubic. Let us first review some intuition for why fast triangle detection should not imply fast matrix multiplication, then discuss how our approach circumvents it. For simplicity, let us focus on the case of Boolean matrix multiplication (BMM) over OR and AND.

First, note that triangle detection returns one bit, while BMM returns $n^{2}$ bits. This seems to indicate that $O\left(n^{2.99}\right)$ triangle detection would be useless for subcubic BMM, as the algorithm would need to be run $\Omega\left(n^{2}\right)$ times. Furthermore, BMM can determine for all edges if there is a triangle using the edge, while triangle detection only determines if some edge is in a triangle. Given our intuitions about quantifiers, it looks unlikely that the universally quantified problem could be efficiently reduced to the existentially quantified problem. So there appears to be strong intuition for why such a reduction would not be possible.

However, there is an advantage to be found in calling triangle detection on small graphs corresponding to small submatrices. Let $A$ and $B$ be $n \times n$ matrices over $\{0,1\}$. Observe that triangle detection can tell us if $A \cdot B$ contains any entry with a 1: Set up a tripartite graph with parts $S_{1}, S_{2}$ and $S_{3}$, each containing $n$ nodes which we identify with $[n]$. The edge relation for $S_{1} \times S_{2}$ is defined by $A$, and the edge relation for $S_{2} \times S_{3}$ is defined by $B$ (in the natural way). A path of length two from $i \in S_{1}$ to $j \in S_{3}$ corresponds to a 1 in the entry $(A \cdot B)[i, j]$. Putting all possible edges between $S_{1}$ and $S_{3}$, there is a triangle in this graph if and only if $A \cdot B$ contains a 1-entry. (Note we are already relying on the fact that our addition operation is OR.)

The above reasoning can also be applied to submatrices $A^{\prime}$ and $B^{\prime}$, to determine if $A^{\prime} \cdot B^{\prime}$ contributes a 1-entry to the matrix product. More generally, triangle detection can tell us if a product of two submatrices contains a 1-entry, among just those entries of the product that we have not already computed. That is, we only need to put edges between $S_{1}$ and $S_{3}$ that correspond to undetermined entries of the product. That is, 
triangle detection can tell us if submatrices $A^{\prime}$ and $B^{\prime}$ have any new 1-entries to contribute to the current matrix product so far.

On the one hand, if all possible pairs of submatrices from $A$ and $B$ do not result in finding a triangle, then we have computed all the 1-entries and the rest must be zeroes. On the other hand, when we detect a triangle, we determine at least one new 1-entry $(i, j)$ in $A \cdot B$, and we can keep latter triangle detection calls from recomputing this entry by removing the edge $(i, j)$ between $S_{1}$ and $S_{3}$. By balancing the number of triangle detection subproblems we generate with the number of 1-entries in $A \cdot B$, we get a subcubic runtime for matrix multiplication provided that the triangle algorithm was also subcubic. (In fact we get an output sensitive algorithm.) With additional technical effort and a simultaneous binary search method, the above ideas can be generalized to any matrix product where "addition" is a minimum operator.

\section{B Prior work}

Matrix Products and Path Problems. Matrix multiplication is fundamental to computer science. The case of multiplying over a ring is well known to admit surprisingly faster algorithms using the magic of subtraction, beginning with the famous algorithm of Strassen [Str69] showing that it is in $O\left(n^{\log _{2} 7}\right)$ time. After many improvements on Strassen's original result, the current best upper bound on ring matrix multiplication is $O\left(n^{2.376}\right)$ by Coppersmith and Winograd [CW90].

Over algebraic structures without subtraction, there has been little progress in the search for truly subcubic algorithms. These "exotic" matrix products are extremely useful in graph algorithms and optimization. For example, matrix multiplication over the ( $\max , \min )$-semiring, with max and min operators in place of plus and times (respectively), can be used to solve the all pairs bottleneck paths problem (APBP) on arbitrary weighted graphs, where we wish to find a maximum capacity path from $s$ to $t$ for all pairs of nodes $s$ and $t$. Recent work [VWY07, DP09] has shown that fast matrix multiplication over rings can be applied to obtain a truly subcubic algorithm over the (max, min)-semiring, yielding truly subcubic APBP. Matrix multiplication over the (min, +)-semiring (also known as the distance product) can be used to solve all pairs shortest paths (APSP) in arbitrary weighted graphs [FM71]. That is, truly subcubic distance product would imply truly subcubic APSP, one of the "Holy Grails" of graph algorithms. The fastest known algorithms for distance product are the $O\left(n^{3} \log \log ^{3} n / \log ^{2} n\right)$ solution due to Chan [Cha05], and $\tilde{O}\left(M n^{\omega}\right)$ where $M$ is the largest weight in the matrices due to Alon, Galil and Margalit [AGM97] (following Yuval [Yuv76]). Unfortunately, the latter is pseudopolynomial (exponential in the bit complexity), and can only be used to efficiently solve APSP in special cases [SZ99].

Many over the years have asked if APSP can be solved faster than cubic time. For an explicit reference, Shoshan and Zwick [SZ99] asked if the distance product of two $n \times n$ matrices with entries in $\{1, \ldots, M\}$ can be computed in $O\left(n^{3-\delta} \log M\right)$ for some $\delta>0$. (Note an APSP algorithm of similar runtime would follow from such an algorithm.)

Triangles and Matrix Products. Itai and Rodeh [IR78] were the first to show that triangle detection can be done with Boolean matrix multiplication.

The trilinear decomposition of Pan [Pan78, Pan80] implies that any bilinear circuit for computing the trace of the cube of a matrix $A$ (i.e., $\operatorname{tr}\left(A^{3}\right)$ ) over any ring can be used to compute matrix products over any ring. So in a sense, algebraic circuits that can count the number of triangles in a graph can be turned into matrix multiplication circuits. Note, this correspondence relies heavily on the algebraic circuit model: it is non-black box in an extreme way. (Our reductions are all black box.) 
The $k$ Shortest Paths and Replacement Paths Problems. A natural generalization of the $s, t$-shortest path problem is that of returning the first $k$ of the shortest paths between $s$ and $t$. In the early 1970s, Yen [Yen71] and Lawler [Law72] presented an algorithm which solved this problem for directed graphs with $m$ edges, $n$ nodes and nonnegative edge weights; with Fibonacci heaps [FT87] their algorithm runs in $O\left(k\left(m n+n^{2} \log n\right)\right)$ time. Eppstein [Epp98] showed that if the paths can have cycles, then the problem can be solved in $O(k+m+n \log n)$ time. When the input graph is undirected, even the $k$ shortest simple paths problem is solvable in $O(k(m+n \log n))$ time [KIM82]. For directed unweighted graphs, the best known algorithm for the problem is the $\tilde{O}(k m \sqrt{n})$ time randomized combinatorial algorithm of Roditty and Zwick [RZ05]. Roditty [Rod07] noticed that the $k$ shortest simple paths can be approximated fast, culminating in Bernstein's [Ber10] amazing $\tilde{O}(\mathrm{~km} / \varepsilon)$ running time for a $(1+\varepsilon)$-approximation. When the paths are to be computed exactly, however, the best running time is still the $O\left(k\left(m n+n^{2} \log n\right)\right)$ time of Yen and Lawler's algorithm.

Roditty and Zwick [RZ05] showed that the $k$ shortest simple paths can be reduced to $k$ computations of the second shortest simple path, and so any $T(m, n)$ time algorithm for the second shortest simple path implies an $O(k T(m, n))$ algorithm for the $k$ shortest simple paths. The second shortest simple path always has the following form: take a prefix of the shortest path $P$ to some node $x$, then take a path to some node $y$ on $P$ using only edges that are not on $P$ (this part is called a detour), then take the remaining portion of $P$ to $t$. The problem then reduces to finding a good detour.

A related problem is the so called replacement paths problem in which one is required to find for every edge $e$ on the shortest path from $s$ to $t$ in $G$, the shortest path between $s$ and $t$ in $G \backslash\{e\}$. The replacement paths problem is at least as hard as the second shortest simple path problem, and so the best known algorithms for it in directed graphs also take $O\left(m n+n^{2} \log n\right)$ time.

Verifying a Metric. In the metricity problem, we are given an $n \times n$ matrix and want to determine whether it defines a metric on $[n]$. The metricity problem is a special case of the metric nearness problem (MNP): given a matrix $D$, find a closest matrix $D^{\prime}$ such that $D$ dominates $D^{\prime}$ and $D^{\prime}$ satisfies the triangle inequality. Brickell et. al [BDST08] show that MNP is equivalent to APSP and ask whether the metricity problem is equivalent to MNP. Theorem 3.2 partially answers their question in the sense that subcubic metricity implies subcubic MNP.

Prior reductions of APSP to other problems. Roditty and Zwick [RZ04] consider the incremental and decremental versions of the single source shortest path problem in weighted and unweighted directed graphs. They show that either APSP has a truly subcubic algorithm, or any data structure for the decremental/incremental single source shortest paths problem must either have been initialized in cubic time, or its updates must take amortized $\Omega\left(n^{2}\right)$ time, or its query time must be $\Omega(n)$. They also give a similar relationship between the problem for unweighted directed graphs and combinatorial algorithms for BMM.

\section{Subcubic Reducibility}

Here we formally define the notion of subcubic reducibility used in this paper, and prove a few consequences of it. Recall that an algorithm with oracle access to $B$ has special workspace in memory reserved for oracle calls, and at any step in the algorithm, it can call $B$ on the content of the special workspace in one unit of time and receive a solution to $B$ in the workspace.

Let $\Sigma$ be an underlying alphabet. We define a size measure to be any function $m: \Sigma^{\star} \rightarrow \mathbb{N}$. In this paper, the size measure on weighted graphs with weights from $[-M, M]$ (or square matrices with entries 
from $[-M, M]$ ) is taken to be the number of nodes in the given graph times $\log M$ (or the matrix dimension times $\log M)$.

Definition C.1 Let $A$ and $B$ be computational problems with a common size measure $m$ on inputs. We say that there is a subcubic reduction from $A$ to $B$ if there is an algorithm $\mathcal{A}$ with oracle access to $B$, such that for every $\varepsilon>0$ there is a $\delta>0$ satisfying three properties:

- For every instance $x$ of $A, \mathcal{A}(x)$ solves the problem $A$ on $x$.

- $\mathcal{A}$ runs in $O\left(m^{3-\delta}\right)$ time on instances of size $m$.

- For every instance $x$ of $A$ of size $m$, let $m_{i}$ be the size of the ith oracle call to $B$ in $\mathcal{A}(x)$. Then $\sum_{i} m_{i}^{3-\varepsilon} \leq m^{3-\delta}$.

We use the notation $A \leq_{3} B$ to denote the existence of a subcubic reduction from $A$ to $B$, and $A \equiv_{3} B$ as shorthand for $A \leq_{3} B$ and $B \leq_{3} A$. In such a case we say that $A$ and $B$ are subcubic-equivalent.

There is a natural extension of the concept to $O\left(n^{q}\right)$ running times, for any constant $q \geq 1$, by replacing all occurrences of 3 in the above definition with $q$. For such reductions we denote their existence by $A \leq_{q} B$, and say there is a sub-q reduction from $A$ to $B$, for values of $q$ such as "quadratic", "cubic", "quartic", etc.

First let us observe that the reducibility relation is transitive.

Proposition 1 Let $A, B, C$ be problems so that $A \leq_{q} B$ and $B \leq_{q} C$. Then $A \leq_{q} C$.

Proof. By definition, we have:

1. For every $\varepsilon>0$ there exists a $\delta>0$ so that for large enough $n$ there exist $\left\{n_{i}\right\}$ with $\sum_{i} n_{i}^{q-\varepsilon} \leq n^{q-\delta}$ and an algorithm $P_{A, \varepsilon}$ for $A$ which on instances of size $n$ runs in $O\left(n^{q-\delta}\right)$ time and makes oracle calls to $B$ with sizes $n_{i}$.

2. For every $\varepsilon^{\prime}>0$ there exists a $\delta^{\prime}>0$ so that for all large enough $n_{i}$ there exist $\left\{n_{i j}\right\}$ with $\sum_{j} n_{i j}^{q-\varepsilon^{\prime}} \leq$ $n_{i}^{q-\delta^{\prime}}$ and an algorithm $P_{B, \varepsilon^{\prime}}$ for $B$ which on instances of size $n_{i}$ runs in $O\left(n_{i}^{q-\delta^{\prime}}\right)$ time and makes oracle calls to $C$ with sizes $n_{i j}$.

We will show that:

3. For every $\varepsilon^{\prime}>0$ there exists a $\delta^{\prime \prime}>0$ so that for all large enough $n$ there exist $\left\{n_{i j}\right\}$ with $\sum_{i j} n_{i j}^{q-\varepsilon^{\prime}} \leq$ $n^{q-\delta^{\prime \prime}}$ and an algorithm $P_{\varepsilon^{\prime}}$ for $A$ which on instances of size $n$ runs in $O\left(n^{q-\delta^{\prime \prime}}\right)$ time and makes oracle calls to $C$ with sizes $n_{i j}$.

Let $\varepsilon^{\prime}>0$ be given. Consider $P_{B, \varepsilon^{\prime}}$ and let $\delta^{\prime}>0$ be the value corresponding to $\varepsilon^{\prime}$, as in 2. Pick $\varepsilon=\delta^{\prime}$. Consider algorithm $P_{A, \varepsilon}$ and let $\delta>0$ be the value corresponding to $\varepsilon$, as in 1 . Replace each oracle call from algorithm $P_{A, \varepsilon}$ for size $n_{i}$ with a call to $P_{B, \varepsilon^{\prime}}$.

Now, the new algorithm $P_{\varepsilon^{\prime}}$ makes oracle calls to $C$ of sizes $n_{i j}$ and runs in time

$$
O\left(n^{q-\delta}+\sum_{i} n_{i}^{q-\delta^{\prime}}\right)
$$

As we picked $\varepsilon=\delta^{\prime}, \sum_{i} n_{i}^{q-\delta^{\prime}}=\sum_{i} n_{i}^{q-\varepsilon} \leq n^{q-\delta}$ (from 1), and the runtime of $P_{\varepsilon^{\prime}}$ is $O\left(n^{q-\delta}\right)$. 
Consider the oracle calls. They are of sizes $\left\{n_{i j}\right\}$ so that, as in 2 , for each $i, \sum_{j} n_{i j}^{q-\varepsilon^{\prime}} \leq n_{i}^{q-\delta^{\prime}}$. Hence

$$
\sum_{i j} n_{i j}^{q-\varepsilon^{\prime}} \leq \sum_{i} n_{i}^{q-\delta^{\prime}}=\sum_{i} n_{i}^{q-\varepsilon} \leq n^{q-\delta}
$$

where the last inequality is from 1 . We can set $\delta^{\prime \prime}=\delta$ and so $A \leq_{q} C$.

Now let us verify that the definition gives us the property we want. In the following, let $A$ and $B$ be computational problems on $n \times n$ matrices with entries in $[-M, M]$ (or equivalently, weighted graphs on $n$ nodes).

Proposition 2 If $A \leq_{3} B$ then a truly subcubic algorithm for $B$ implies a truly subcubic algorithm for $A$.

Proof. If there is an $O\left(n^{3-\varepsilon}\right.$ poly $\left.\log M\right)$ algorithm for $B$ then the algorithm for $A$ in the reduction runs in $\sum_{i} n_{i}^{3-\varepsilon}$ poly $\log M \leq n^{3-\delta}$ poly $\log M$ time.

Strongly Subcubic Reductions. All subcubic equivalences proved in this paper have one additional property in their reductions: the number of oracle calls and the sizes of oracle calls depend only on the input, and not on the parameter $\varepsilon$. (In some other reductions, such as the example below, this is not the case.) Let us define a reduction with this property to be a strongly subcubic reduction. These stronger reductions have the nice quality that, with respect to polylogarithmic improvements, running times are preserved.

Theorem C.1 If there is a strongly subcubic reduction from $A$ to $B$, then

- For all $c>0$, an $O\left(n^{3}(\log M)^{d} / \log ^{c} n\right)$ algorithm for $B$ implies an $O\left(n^{3}(\log M)^{3 d} / \log ^{c} n\right)$ algorithm for $A$, and an $O\left(n^{3} / \log ^{c} n\right)$ algorithm for $B$ implies an $O\left(n^{3} / \log ^{c} n\right)$ algorithm for $A$.

- For all $\gamma>0$, an $n^{3} / 2^{\Omega\left(\log ^{\gamma} n\right)}$ algorithm for B implies an $n^{3} / 2^{\Omega\left(\log ^{\gamma} n\right)}$ algorithm for $A$.

Proof. For simplicity let $n$ be the input size measure. First, we show that

$$
\sum_{i} n_{i}^{3} \leq n^{3}
$$

A strongly subcubic reduction gives us a fixed algorithm such that for all sizes $n$, the number of oracle calls and the sizes of oracle calls $\left\{n_{i}\right\}$ depend only on the input. Then, for all $\varepsilon>0$, there is a $\delta>0$ satisfying

$$
\sum_{i} n_{i}^{3-\varepsilon} \leq n^{3-\delta}<n^{3}
$$

Since $\left\{n_{i}\right\}$ and $n$ are independent of $\varepsilon$, this means that for every fixed set $\left\{n_{i}\right\}$ and $n$, we can take the limit on both sides of the above inequality as $\varepsilon \rightarrow 0$. We obtain that for every $n$ and every set of oracle call sizes $\left\{n_{i}\right\}$ on an input of size $n, \sum_{i} n_{i}^{3} \leq n^{3}$.

Now consider an algorithm for $B$ that runs in $O\left(n^{3} / \log ^{c} n\right)$ time. Then an algorithm for $A$ that uses the reduction calling $B$ as an oracle would run in $O\left(n^{3-\delta}+\sum_{i} n_{i}^{3} / \log ^{c} n_{i}\right)$ time for some $\delta>0$. Let $a<\delta / 3$. Then

$$
\sum_{i} n_{i}^{3} / \log ^{c} n_{i}=\sum_{i: n_{i}<n^{a}} n_{i}^{3} / \log ^{c} n_{i}+\sum_{i: n_{i} \geq n^{a}} n_{i}^{3} / \log ^{c} n_{i}
$$


which is at most

$$
O\left(n^{3-\delta} \cdot n^{3 a}+\sum_{i: n_{i} \geq n^{a}} n_{i}^{3} / \log ^{c} n\right)
$$

since the number of oracle calls is at most $O\left(n^{3-\delta}\right)$. The first term is $n^{3-\varepsilon^{\prime}}$ for some $\varepsilon^{\prime}>0$, by our choice of a. By (2), we have

$$
O\left(n^{3-\varepsilon^{\prime}}+\sum_{i: n_{i} \geq n^{a}} n_{i}^{3} / \log ^{c} n\right) \leq O\left(n^{3} / \log ^{c} n\right) .
$$

The proof of the second item is analogous.

It can be shown that strongly subcubic reductions are necessary for Theorem $\mathrm{C} .1$ to hold. If the sizes of oracle calls or their number depend on $\varepsilon$, one can find cases where polylog factors are diminished in the algorithm for $A$. (In fact, the reduction below of Matoušek is one example.)

These kinds of reductions were implicit in prior work, but have not been studied systematically. For one example, Matoušek [Mat91] showed that computing dominances in $\mathbb{R}^{n}$ between pairs of $n$ vectors can be done in $O\left(n^{(3+t) / 2}\right)$ time, where $O\left(n^{t}\right)$ is an upper bound on $n \times n$ integer matrix multiplication. The algorithm works by making $O\left(n^{3 / 2} / n^{t / 2}\right)$ calls to $n \times n$ integer matrix multiplication. (Note this is not a strongly subcubic reduction, since the number of calls depends on $t$.) Notice that for any $t<3$, the running time $O\left(n^{(3+t) / 2}\right)$ is truly subcubic. Hence we can say:

\section{Dominances in $\mathbb{R}^{n} \leq_{3}$ Integer Matrix Multiplication.}

Another example is that of 3SUM-hardness in computational geometry. Gajentaan and Overmars [GO95] showed that for many problems $\Pi$ solvable in quadratic time, one can reduce 3SUM to $\Pi$ in such a way that a subquadratic algorithm for $\Pi$ implies one for 3SUM. Hence under the conjecture that the 3SUM problem is hard to solve faster, many other $\Pi$ are also hard 6 Proofs of 3 SUM-hardness imply 3 SUM $\leq_{2} \Pi$, but the notion of reduction used in [GO95] is weaker than ours. (They only allow $O(1)$ calls to the oracle for $\Pi$.)

\section{Proofs of the generic results on detection, finding, listing}

Reminder of Lemma 3.1 (Folklore) Let $T(n)$ be a function so that $T(n) / n$ is nondecreasing. If there is a $T(n)$ time algorithm for negative triangle detection over $\mathcal{R}$ on a graph $G=(I \cup J \cup K, E)$, then there is an $O(T(n))$ algorithm which returns a negative triangle over $\mathcal{R}$ in $G$ if one exists.

Proof of Lemma 3.1. The algorithm is recursive: it proceeds by first splitting $I, J$ and $K$ each into two roughly equal parts $I_{1}$ and $I_{2}, J_{1}$ and $J_{2}$, and $K_{1}$ and $K_{2}$. Then it runs the detection algorithm on all 8 induced subinstances $\left(I_{i}, J_{j}, K_{k}\right), i, j, k \in\{1,2\}$. If none of these return 'yes', then there is no negative triangle in $G$. Otherwise, the algorithm recurses on exactly one subinstance on which the detection algorithm returns 'yes'. The base case is when $|I|=|J|=|K|=1$ and then one just checks whether the three nodes form a triangle in $O(1)$ time. The running time becomes

$$
T^{\prime}(n)=8 T(n)+T^{\prime}(n / 2), T^{\prime}(1)=1 .
$$

\footnotetext{
${ }^{6}$ Sometimes $\Pi$ is defined to be 3 SUM-hard if " $\Pi$ is in subquadratic time implies 3SUM is in subquadratic time". This definition leaves something to be desired: if 3SUM turned out to be in subquadratic time then all problems are 3SUM-hard, and if 3SUM is not in subquadratic time then no subquadratic problem is 3SUM-hard. Hence the 3SUM-hardness of some problems would be contingent on the complexity of 3SUM itself. Note this is not the definition of [GO95], which is a reducibility notion like ours.
} 
If $T(n)=n f(n)$ for some nondecreasing function $f(n)$, then $T(n)=2 \frac{n}{2} f(n) \geq 2 \frac{n}{2} f(n / 2)=2 T(n / 2)$. Hence the recurrence above solves to $T^{\prime}(n)=O(T(n))$.

Reminder of Theorem 3.3 Suppose there is a truly subcubic algorithm for negative triangle detection over $\mathcal{R}$. Then there is a truly subcubic algorithm which lists $\Delta$ negative triangles over $\mathcal{R}$ in any graph with at least $\Delta$ negative triangles, for any $\Delta=O\left(n^{3-\delta}\right), \delta>0$.

Proof of Theorem 3.3. Let $P$ be an $O\left(n^{3-\varepsilon} \log ^{c} M\right)$ algorithm for negative triangle over $\mathcal{R}$ for $\varepsilon>0$. Let $\Delta=O\left(n^{3-\delta}\right)$ for $\delta>0$. Given an $3 n$-node tripartite graph $G=(I \cup J \cup K, E)$ with at least $\Delta$ negative triangles over $\mathcal{R}$ we provide a procedure to list $\Delta$ negative triangles over $\mathcal{R}$.

We partition the nodes in $I, J, K$ into $\Delta^{1 / 3}$ parts, each of size $O\left(n / \Delta^{1 / 3}\right)$. For all $\Delta$ triples $I^{\prime} \subset I, J^{\prime} \subset$ $J, K^{\prime} \subset K$ of parts, run $P$ in $O\left(\Delta\left(n / \Delta^{1 / 3}\right)^{3-\varepsilon} \log ^{c} M\right)$ time overall to determine all triples which contain negative triangles over $\mathcal{R}$.

On the triples which contain negative triangles, we run a recursive procedure. Let $I^{\prime} \subset I, J^{\prime} \subset J, K^{\prime} \subset K$ be a triple which is reported to contain a negative triangle over $\mathcal{R}$. Split $I^{\prime}, J^{\prime}$ and $K^{\prime}$ each into two roughly equal halves. On each of the 8 possible triples of halves, run $P$ and recurse on the triples of halves which contain negative triangles, with the following provision. For each level $i$ of recursion (where $i$ ranges from 0 to $\left.\log \frac{n}{\Delta^{1 / 3}}\right)$, we maintain a global counter $c_{i}$ of the number of recursive calls that have been executed at that level. Once $c_{i}>\Delta$ then we do not recurse on any more triples at recursion level $i$. Once a triple only contains 3 nodes, we output it if it forms a negative triangle. Notice that all listed triangles are distinct.

Level $i$ of the recursion examines triples which contain $O\left(\frac{n}{2^{i} \Delta^{1 / 3}}\right)$ nodes. At each level $i$, at most $\Delta$ triples containing negative triangles are examined, due to the global counters. Therefore the runtime at level $i$ is at most $O\left(\Delta \cdot\left(\frac{n}{2^{i} \Delta^{1 / 3}}\right)^{3-\varepsilon} \log ^{c} M\right)$. Since $\varepsilon<3$, the overall runtime becomes asymptotically

$$
\Delta\left(\frac{n}{\Delta^{1 / 3}}\right)^{3-\varepsilon} \log ^{c} M \cdot \sum_{i}\left(\frac{1}{2^{3-\varepsilon}}\right)^{i}=O\left(\Delta^{\varepsilon / 3} n^{3-\varepsilon} \log ^{c} M\right) .
$$

When $\Delta \leq O\left(n^{3-\delta}\right)$, the runtime is

$$
O\left(n^{3-\varepsilon+3 \varepsilon / 3-\delta \varepsilon / 3} \log ^{c} M\right)=O\left(n^{3-\delta \varepsilon / 3} \log ^{c} M\right),
$$

which is truly subcubic for any $\varepsilon, \delta>0$.

\section{E Proof of Theorem 3.4}

Theorem 3.4 follows from a more general lemma:

Lemma E.1 Let $T(n)$ be a function so that $T(n) / n$ is nondecreasing. Suppose there is a $T(n)$ time algorithm for negative triangle detection over $\mathcal{R}$ in an n node graph. Then:

- There is an algorithm that computes $\ell$ entries of the product over $\mathcal{R}$ of an $m \times n$ matrix by an $n \times p$ matrix in $O\left(\ell \cdot T\left((m n p / \ell)^{1 / 3}\right) \log W\right)$ time, where the entries in the output lie in $[-W, W] \cup\{-\infty, \infty\}$.

- If there is a constant $\varepsilon: 0<\varepsilon<1$ such that for all large enough $n, T(n) \geq T\left(2^{1 / 3} n\right) /(2(1-\varepsilon))$, then there is an $O\left(\ell \cdot T\left((m n p / \ell)^{1 / 3}\right)\right)$-time algorithm for computing the product over $\mathcal{R}$ of an $m \times n$ by an $n \times p$ matrix, where $\ell$ is the number of ones in the product matrix. 
Proof. Following the ideas from Theorem $3.2, \ell I J$-disjoint negative triangles over $\mathcal{R}$ can be found in

$$
O\left(\left(\ell+a^{3}\right) \cdot T\left((m n p)^{1 / 3} / a\right)\right)
$$

time, where $a$ is a bucketting parameter. We set $a=\ell^{1 / 3}$ and we get a runtime of $O\left(\ell \cdot T\left((m n p / \ell)^{1 / 3}\right)\right)$. We set $\ell=m p$ and apply binary search on top of this to obtain a matrix product algorithm. To get an output-sensitive algorithm, for each $i=1, \ldots, \log m p$, we set $a=2^{i / 3}$; the runtime is now

$$
\sum_{i=1}^{\log m p} 2^{i} \cdot T\left(\left(m n p / 2^{i}\right)^{1 / 3}\right) .
$$

Since there is a constant $\varepsilon<1$ so that for all $n, T(n) \geq T\left(2^{1 / 3} n\right) /(2(1-\varepsilon))$, then for all $i, 2^{i} T\left((m n p)^{1 / 3} / 2^{i / 3}\right) \leq$ $2^{i+1}(1-\varepsilon) T\left((m n p)^{1 / 3} / 2^{(i+1) / 3}\right)$ and hence the runtime is $O\left(\ell \cdot T\left((m n p / \ell)^{1 / 3}\right)\right)$, where $\ell$ is the number of ones in the output.

\section{F Problems Equivalent to All-Pairs Shortest Paths}

The goal of this section is to prove Theorem 1.1 from the Introduction.

Reminder of Theorem 1.1 The following weighted problems either all have truly subcubic algorithms, or none of them do:

1. The all-pairs shortest paths problem on weighted digraphs (APSP).

2. Detecting if a weighted graph has a triangle of negative total edge weight.

3. Listing up to $n^{2.99}$ negative triangles in an edge-weighted graph.

4. Verifying the correctness of a matrix product over the (min, + )-semiring.

5. The all-pairs shortest paths problem on undirected weighted graphs.

6. Checking whether a given matrix defines a metric.

7. Finding a minimum weight cycle in a graph of non-negative edge weights.

8. The replacement paths problem on weighted digraphs.

9. Finding the second shortest simple path between two nodes in a weighted digraph.

The subcubic equivalence of $1,2,3$, and 4 directly follow from Theorems 3.1, 3.2 and 3.3. The rest of the equivalences are proved in the following. Most of our equivalences use the negative triangle problem, since it is so easy to reason about.

The equivalence between 1 and 5 is probably folklore, but we have not seen it in the literature so we include it for completeness.

Theorem F.1 (Undirected APSP $\equiv_{3}$ Directed APSP) Let $\delta, c>0$ be any constants. APSP in undirected graphs with weights in $[-M, M]$ is in $\tilde{O}\left(n^{3-\delta} \log ^{c} M\right)$ time iff APSP in directed graphs with weights in $[-M, M]$ is in $\tilde{O}\left(n^{3-\delta} \log ^{c} M\right)$ time.

Proof of Theorem F.1 Clearly, undirected APSP is a special case of directed APSP. We show that a truly subcubic algorithm for undirected APSP can be used to compute the $(\mathrm{min},+)$ product of two matrices in truly subcubic time, and hence directed APSP is in truly subcubic time. 
Suppose that there is a truly subcubic algorithm $P$ for undirected APSP. Let $A$ and $B$ be the $n \times n$ matrices whose $(\mathrm{min},+)$ product we want to compute. Suppose the entries of $A$ and $B$ are in $[-M, M]$. Consider the edge-weighted undirected tripartite graph $G$ with $n$-node partitions $I, J, K$ such that there are no edges between $I$ and $K$, and for all $i \in I, j \in J, k \in K,(i, j)$ and $(j, k)$ are edges with $w(i, j)=A[i, j]+6 M$ and $w(j, k)=B[j, k]+6 M$. Using $P$, compute APSP in $G$.

Any path on at least 3 edges in $G$ has weight at least $15 M$, and any path on at most 2 edges has weight at most $2 \times 7 M<15 M$. Hence $P$ will find for every two nodes $i \in I, k \in K$, the shortest path between $i$ and $k$ using exactly 2 edges, thus computing the $(\min ,+)$ product of $A$ and $B$.

Theorem F.2 (Metricity $\equiv_{3}$ Negative Triangle) Let $T(n, M)$ be nondecreasing. Then there is an $O\left(n^{2}\right)+$ $T(O(n), O(M))$ algorithm for negative triangle in $n$ node graphs with weights in $[-M, M]$ if and only if there is an $O\left(n^{2}\right)+T(O(n), O(M))$ algorithm for the metricity problem on $[n]$ such that all distances are in $[-M, M]$.

Proof of Theorem F.2. Given an instance $D$ of the metricity problem, consider a complete tripartite graph $G$ on $3 n$ nodes $n$ nodes in each of the partitions $I, J, K$. For any $i \in I, j \in J, k \in K$, define the edge weights to be $w(i, j)=D[i, j], w(j, k)=D[j, k]$ and $w(i, k)=-D[i, k]$. A negative triangle in $G$ gives $i \in I, j \in J, k \in K$ so that $D[i, j]+D[j, k]-D[i, k]<0$, i.e. $D[i, j]+D[j, k]<D[i, k]$. Hence $D$ satisfies the triangle inequality iff there are no negative triangles in $G$. Checking the other properties for a metric takes $O\left(n^{2}\right)$ time.

Let $G$ be a given a graph with edge weights $w: E \rightarrow \mathbb{Z}$ which is an instance of negative triangle so that for all $e \in E, w(e) \in[-M, M]$ for some $M>0$. Build a tripartite graph with $n$ node partitions $I, J, K$ and edge weights $w^{\prime}(\cdot)$ so that for any $i \in I, j \in J, k \in K, w^{\prime}(i, j)=2 M+w(i, j), w^{\prime}(j, k)=2 M+w(j, k)$ and $w^{\prime}(i, k)=4 M-w(i, k)$. For all pairs of distinct nodes $a, b$ so that $a, b$ are in the same partition, let $w^{\prime}(a, b)=2 M$. Finally, let $w^{\prime}(x, x)=0$ for all $x$. Clearly, $w^{\prime}$ satisfies all requirements for a metric except possibly the triangle inequality. For any three vertices $x, y, z$ in the same partition $w^{\prime}(x, y)+w^{\prime}(y, z)=$ $4 M>2 M=w^{\prime}(x, z)$. Consider triples $x, y, z$ of vertices so that $x$ and $y$ are in the same partition and $z$ is in a different partition. We have: $w^{\prime}(x, z)+w^{\prime}(z, y) \geq M+M=2 M=w^{\prime}(x, y)$ and $w^{\prime}(x, z)-w^{\prime}(y, z) \leq$ $2 M=w^{\prime}(x, y)$. Furthermore, if $i \in I, j \in J, k \in K, w^{\prime}(i, k)+w^{\prime}(k, j) \geq M+3 M \geq w(i, j)$ and $w^{\prime}(i, j)+w^{\prime}(j, k) \geq M+3 M \geq w(i, k)$. Hence the only possible triples which could violate the triangle inequality are triples with $i \in I, j \in J, k \in K$, and $w^{\prime}$ is not a metric iff there exist $i \in I, j \in J, k \in K$ such that $w^{\prime}(i, j)+w^{\prime}(j, k)<w^{\prime}(i, k)$, i.e. $w(i, j)+w(j, k)+w(i, k)<0$ and $i, j, k$ is a negative triangle in $G$.

Theorem F.3 (Minimum Cycle $\equiv_{3}$ Negative Triangle) If there is a $T(n, M)$ algorithm for finding a minimum weight cycle in graphs on $n$ nodes and weights in $[1, M]$ then there is a $T(n, O(M))$ algorithm for finding a minimum weight triangle in n-node graphs with weights in $[-M, M]$.

Since any algorithm for APSP can find a minimum cycle in a graph with nonnegative weights in the same running time, we get that APSP and Minimum-Cycle are equivalent w.r.t. subcubic algorithms.

Proof. Let $G=(V, E)$ be given with $w: E \rightarrow[-M, M]$. Consider graph $G^{\prime}$ which is just $G$ with weights $w^{\prime}: E \rightarrow[7 M, 9 M]$ defined as $w^{\prime}(e)=w(e)+8 M$. For any $k$ and any cycle $C$ in $G$ with $k$ edges, $w^{\prime}(C)=8 M k+w(C)$, and hence $7 M k \leq w^{\prime}(C) \leq 9 M k$. Hence, all cycles $C$ with $\geq 4$ edges have $w^{\prime}(C) \geq 28 M$ and all triangles have $w^{\prime}$ weight $\leq 27 M<28 M$. That is, the minimum weight cycle in $G^{\prime}$ is exactly the minimum weight triangle in $G$.

${ }^{7}$ Infinite edge weights can be replaced with suitably large finite values, WLOG. 
It remains to show the equivalences of 8 and 9 with the other problems.

\section{F.1 Replacement Paths and Second Shortest Paths}

The replacement paths and second shortest simple path problems have been known to be closely related to APSP in an informal sense. For instance, any algorithm for APSP can solve the two problems in asymptotically the same time: remove all edges from the shortest path $P$ between $s$ and $t$ and compute APSP in the remaining graph. This computes the minimum weight detour for all pairs of nodes on $P$, and so in additional $O\left(n^{2}\right)$ time one can solve both the replacement paths problem, and the second shortest simple path problem. It was not clear however that the two problems cannot be solved faster than APSP. For instance, Roditty [Rod07] took his fast approximation algorithms as evidence that the two problems might be easier than APSP. In an attempt to explain why it has been so hard to find fast algorithms, Hershberger et al. [HSB07] showed that in the path comparison model of Karger et al. [KKP93] the replacement paths problem needs $\Omega(m \sqrt{n})$ time. This bound does not apply to second shortest path, and is the only known lower bound for these problems.

Here we present a reduction which shows that if the second shortest simple path in a directed graph with $n$ nodes can be found in time which is truly subcubic in $n$, then APSP is in truly subcubic time. Thus, the two problems are equivalent w.r.t. subcubic algorithms, for dense graphs. Since the second shortest simple path problem is a special case of the replacement paths problem, our result implies that for dense graphs the replacement paths problem is equivalent to APSP, w.r.t. subcubic algorithms.

In the next section we modify the reduction to show that if for some $m(n)$ and nondecreasing $f(n)$ there is a combinatorial algorithm which runs in $O(m(n) \sqrt{n} / f(n))$ time and computes the second shortest simple path in unweighted directed graphs, then there is an $O\left(n^{3} / f(n)\right)$ combinatorial algorithm for triangle detection, and hence a corresponding subcubic combinatorial algorithm for BMM. This implies that if there is no truly subcubic combinatorial algorithm for BMM, then in order to improve on the algorithm of Roditty and Zwick [RZ05], one would need to use algebraic techniques.

Theorem F.4 (Minimum Triangle $\equiv_{3}$ Second Shortest Simple Path) Suppose there is a T(n) time algorithm for computing the second shortest simple path in a weighted directed graph with $n$ nodes. Then there is a $T(O(n))$ time algorithm for finding a minimum weight triangle in an $n$ node graph, and hence an $O\left(n^{2} T\left(O\left(n^{1 / 3}\right)\right) \log W\right)$ time algorithm for APSP in n node graphs with weights in $[-W, W]$.

Proof. Let $G$ be an instance of Minimum Triangle. WLOG $G$ has 3 parts $U, V, T$ with no edges within them, and the edges going from $U$ to $V$, from $V$ to $T$ and from $T$ to $U$. Furthermore, WLOG all edge weights are positive (otherwise add a sufficiently large number $Y$ to all edges, so that now the min weight triangle has weight $3 Y+$ its original weight). WLOG also $G$ contains edges between every two nodes $u_{i} \in U, v_{j} \in V$, between any two nodes $v_{j} \in V$ and $t_{k} \in T$ and between any two nodes $t_{k} \in T$ and $u_{i} \in U$ (if some edge isn't there, add it with weight $3 W^{\prime \prime}+1$ where $W^{\prime \prime}$ is the max edge weight in $G$ ). Note that all of these transformations increased the max weight by at most a constant factor.

Now we will reduce any instance of minimum weight triangle to one of finding the second shortest path. First, create a path on $n+1$ nodes, $P=p_{0} \rightarrow p_{1} \rightarrow \ldots \rightarrow p_{n}$. For every edge $\left(p_{i}, p_{i+1}\right)$ in $P$, let it have weight 0 . All other edges in the graph we will construct will be positive and hence $P$ will be the shortest path between $p_{0}$ and $p_{n}$.

Create three parts with $n$ nodes each, $A=\left\{a_{1}, \ldots, a_{n}\right\}, B=\left\{b_{1}, \ldots, b_{n}\right\}, C=\left\{c_{1}, \ldots, c_{n}\right\}$ so that for each $i, j \in[n]$ there's an edge $\left(a_{i}, b_{j}\right)$ with weight $w\left(u_{i}, v_{j}\right)$ (the weight in $\left.G\right)$, and an edge $\left(b_{i}, c_{j}\right)$ with weight $w\left(v_{i}, t_{j}\right)$; that is, we have created a copy of $G$ except that the edges between $T$ and $U$ are removed (no edges between $C$ and $A$ ). 
Let $W^{\prime}$ be the maximum weight in $G$ and let $W=3 W^{\prime}+1$.

Now, for every $j>0$, add an edge from $c_{j}$ to $p_{j}$ with weight $j W$.

For every $i<n$ and any $r \in[n]$, add an edge from $p_{i}$ to $a_{r}$ with weight $(n-i-1) W+w\left(c_{i+1}, a_{r}\right)$. The second shortest path must have the form $p_{0} \rightarrow \ldots \rightarrow p_{s}$ followed by a path of length two from some $a_{i}$ through a node in $B$ to a node $c_{t}$ in $C$ with $t>s$, followed by an edge $\left(c_{t}, p_{t}\right)$ and then $p_{t} \rightarrow \ldots \rightarrow p_{n}$ : we are looking for the shortest detour between a node $p_{s}$ and a node $p_{t}$ on $P$ with $t>s$.

The weight of a detour between $p_{s}$ and $p_{t}$ going through nodes $a_{i}, b_{j}, c_{t}$ is

$$
(n-s-1) W+w\left(c_{s+1}, a_{i}\right)+w\left(a_{i}, b_{j}\right)+w\left(b_{j}, c_{t}\right)+t W .
$$

Claim 1 In the graph we have constructed, any optimal detour must have $t=s+1$.

Proof of claim: Clearly $t>s$. If $t \geq s+2$, then the weight of the detour is at least

$$
(n-s-1+s+2) W+w\left(c_{s+1}, a_{i}\right)+w\left(a_{i}, b_{j}\right)+w\left(b_{j}, c_{t}\right)>(n+1) W .
$$

Consider any detour between $p_{s}$ and $p_{s+1}$, say going through $a_{i}, b_{j}, c_{s+1}$. Its weight is

$$
(n-s-1+s+1) W+w\left(c_{s+1}, a_{i}\right)+w\left(a_{i}, b_{j}\right)+w\left(b_{j}, c_{s+1}\right) \leq n W+W=(n+1) W,
$$

since $W$ is greater than 3times the largest weight in the graph.

Now, the detours between $p_{s}$ and $p_{s+1}$ have weight $n W+w\left(a_{i}, b_{j}\right)+w\left(b_{j}, c_{s+1}\right)+w\left(c_{s+1}, a_{i}\right)$. In particular, the shortest detour between $p_{s}$ and $p_{s+1}$ has weight $n W+$ the minimum weight triangle containing $c_{s+1}$. The second shortest path hence has weight exactly $n W+$ the minimum weight of a triangle in $G$.

Since the second shortest path problem is a special case of the replacement paths problem, we have:

Corollary F.1 If the replacement paths problem is in $T(n)$ time then APSP is in $O\left(n^{2} T\left(O\left(n^{1 / 3}\right)\right) \log M\right)$ time.

Corollary F.2 Replacement Paths $\equiv_{3}$ APSP.

\section{G Boolean Matrix Multiplication and Related Problems}

In this section, we describe several applications of our techniques to the problem of finding fast practical Boolean matrix multiplication algorithms, a longstanding challenge in graph algorithms. (For more background on this problem, see the Preliminaries.)

As a direct consequence of Theorems 3.2, 3.1 and 3.3 we obtain:

Theorem G.1 The following either all have truly subcubic combinatorial algorithms, or none of them do:

\section{Boolean matrix multiplication (BMM).}

2. Detecting if a graph has a triangle.

3. Listing up to $n^{2.99}$ triangles in a graph.

4. Verifying the correctness of a matrix product over the Boolean semiring. 
Theorem G.2 For any constant c the problems listed in Theorem G.1 either all have combinatorial algorithms running in $O\left(n^{3} / \log ^{c} n\right)$ time, or none of them do.

Another immediate corollary of Theorem 3.3 is an efficient triangle listing algorithm:

Corollary G.1 There is an algorithm that, given $\Delta$ and a graph $G$ on $n$ nodes, lists up to $\Delta$ triangles from $G$ in time $O\left(\Delta^{1-\omega / 3} n^{\omega}\right) \leq O\left(\Delta^{0.21} n^{2.38}\right)$.

Note when $\Delta=n^{3}$, one recovers the obvious $O\left(n^{3}\right)$ algorithm for listing all triangles, and when $\Delta=$ $O(1)$, the runtime is the same as that of triangle detection.

\section{G.1 Output-Sensitive BMM}

Lemma E.1 can be applied to show that in the special case of BMM, there is an improved randomized output-sensitive algorithm:

Theorem G.3 Let $T(n)$ be a function so that $T(n) / n$ is nondecreasing. Let $L \geq n \log n$. Suppose there is $a T(n)$ time algorithm for triangle detection in an $n$ node graph. Then there is a randomized algorithm $R$ running in time

$$
\tilde{O}\left(n^{2}+L \cdot T\left(n^{2 / 3} / L^{1 / 6}\right)\right),
$$

so that $R$ computes the Boolean product $C$ of two given $n \times n$ matrices with high probability, provided that $C$ contains at most $L$ nonzero entries. When $T(n)=O\left(n^{\Delta}\right)$ for some $2 \leq \Delta \leq 3$, the runtime becomes $\tilde{O}\left(n^{2 \Delta / 3} L^{1-\Delta / 6}\right)$.

Proof. The algorithm uses ideas from a paper by Lingas [Lin09]. Lingas showed how to reduce, in $O\left(n^{2} \log n\right)$ time, computing the Boolean matrix product of two $n \times n$ matrices to computing $O(\log n)$ Boolean matrix products of an $O(\sqrt{L}) \times n$ by an $n \times O(\sqrt{L})$ matrix and 2 output-sensitive Boolean matrix products of an $O(\sqrt{L}) \times n$ by an $n \times n$ matrix.

Using Lemma E.1 we get an asymptotic runtime of

$$
n^{2} \log n+\log n \cdot L \cdot T\left(n^{1 / 3}\right)+L \cdot T\left(n^{2 / 3} / L^{1 / 6}\right) .
$$

Since $T(n)$ is nondecreasing and since $L \leq n^{2}$, we get that $T\left(n^{2 / 3} / L^{1 / 6}\right) \geq T\left(n^{1 / 3}\right)$ and hence we can bound the runtime by $O\left(\left(n^{2}+L \cdot T\left(n^{2 / 3} / L^{1 / 6}\right)\right) \log n\right)$.

If $T(n)=O\left(n^{\Delta}\right)$ for some $2 \leq \Delta \leq 3$ and $L \geq n$ we have $L \cdot\left(n^{2 / 3} / L^{1 / 6}\right)^{\Delta} \geq n^{2}$. Hence the runtime is just $\tilde{O}\left(n^{2 \Delta / 3} L^{1-\Delta / 6}\right)$.

\section{G.2 Second Shortest Paths and BMM}

Similar to the case of APSP, we can prove a close relationship between BMM and finding the second simple shortest path between two given nodes in an unweighted directed graph. The relationship naturally extends to a relationship between BMM and RPP in unweighted directed graphs. The theorem below shows that in the realm of combinatorial algorithms, Roditty and Zwick's [RZ05] algorithm for the second shortest simple path problem in unweighted directed graphs would be optimal, unless there is a truly subcubic combinatorial algorithm for BMM. Furthermore, any practical improvement of their algorithm would be interesting as it would imply a new practical BMM algorithm.

Theorem G.4 Suppose there exist nondecreasing functions $f(n)$ and $m(n)$ with $m(n) \geq n$, and a combinatorial algorithm which runs in $O(m(n) \sqrt{n} / f(n))$ time and computes the second shortest simple path in any 
given unweighted directed graph with $n$ nodes and $m(n)$ edges. Then there is a combinatorial algorithm for triangle detection running in $O\left(n^{3} / f(n)\right)$ time. If $f(n)=n^{\varepsilon}$ for some $\varepsilon>0$, then there is a truly subcubic combinatorial algorithm for BMM.

Proof. Suppose we are given an instance of triangle detection $G=(V, E)$ where $V$ is identified with $[n]$. Let $L$ be a parameter. Partition $V$ into $n / L$ buckets $V_{b}=\{b L+1, \ldots, b L+L\}$ of size $L$.

We will create $n / L$ instances of the second shortest simple path problem. In instance $b$ (for $b \in\{0, \ldots, n / L-$ 1 ), we will be able to check whether there is a triangle going through a node in bucket $V_{b}$.

Fix some $b$. First, create a path on $L+1$ nodes, $P=p_{0} \rightarrow p_{1} \rightarrow \ldots \rightarrow p_{L}$.

In our construction we will make sure that $P$ is the shortest path from $p_{0}$ to $p_{L}$. The second shortest path would have to go from $p_{0}$ to some $p_{s}$ using $P$, then take a detour (say of length $d$ ) to $p_{t}$ with $t>s$, and then take $P$ from $p_{t}$ to $p_{L}$. The length of the second shortest path would then be

$$
L-t+s+d=L+d+(s-t)
$$

Create three parts, $A=\left\{a_{1}, \ldots, a_{n}\right\}, B=\left\{b_{1}, \ldots, b_{n}\right\}, C=\left\{c_{1}, \ldots, c_{L}\right\}$ so that for each $i, j \in[n]$ there is an edge $\left(a_{i}, b_{j}\right)$ iff $(i, j) \in E$ and for every $i \in[n], j \in[L]$, there is an edge $\left(b_{i}, c_{j}\right)$ iff $(i, b L+j) \in E$.

Now, for every $j>0$, add a path $R_{j}$ of length $2 j$ from $c_{j}$ to $p_{j}$, adding $2 j$ new nodes.

For every $i<L$ add a path $Q_{i}$ of length $2(2 L-i)$, ending at some node $q_{i}$ (thus adding $4 L-2 i$ new nodes). The overall number of new nodes is at most $4 L(L+1)$.

For every $r \in[n]$ and $i<L$, add an edge from $q_{i}$ to $a_{r}$ iff $(b L+i+1, r) \in E$.

Now, any simple path from $p_{0}$ to $p_{L}$ which uses nodes from $A, B$ or $C$ must go through one of the paths $Q_{i}$, and hence has length at least $2(2 L-L+1)=2(L+1)>L+1$. Hence $P$ is the shortest path between $p_{0}$ and $p_{L}$.

The second shortest path must have the form $p_{0} \rightarrow \ldots \rightarrow p_{s}$ followed by a detour to $p_{t}$ for $t>s$, followed by $p_{t} \rightarrow \ldots \rightarrow p_{L}$. The detours between $p_{s}$ and $p_{t}$ look like this: take path $Q_{s}$ from $p_{s}$ to $q_{s}$, then a path of length 3 through some $a_{i}$ through a node in $B$ to a node $c_{t}$ in $C$ with $t>s$, and then taking path $R_{t}$ to $p_{t}$. The length of the detour is

$$
d_{s t}=2(2 L-s)+3+2 t=4 L+3+2(t-s) .
$$

The length of the full path is

$$
L+d_{s t}+(s-t)=5 L+3+(t-s) .
$$

Hence the closer $s$ and $t$ are, the shorter the path.

Now, $G$ has a triangle $(i, j, b L+s)$ going through $V_{b}$ iff there is a path with detour between $p_{s-1}$ and $p_{s}$ going through $Q_{s-1}, a_{i}, b_{j}, c_{s}, R_{s}$. Its length is $5 L+4$. For any $s, t$ with $t \geq s+2$, the length of the path with detour between $p_{s}$ and $p_{t}$ is at least $5 L+3+2>5 L+4$. Hence the shortest that a second shortest path can be is $5 L+4$. It is exactly of this length (and goes between some $p_{s}$ and $p_{s+1}$ ) iff there is a triangle going through $V_{b}$. Computing the length of the second shortest simple path then will tell us whether the original graph has a triangle going through $V_{b}$.

Each of the $n / L$ graphs (for each setting of $b$ ) has $O\left(n+L^{2}\right)$ nodes and $O\left(n^{2}\right)$ edges. For $L=\Omega(\sqrt{n})$ the graph has $O\left(L^{2}\right)$ nodes and $O\left(n^{2}\right)$ edges.

Suppose that for some nondecreasing $m(N)$ and $f(N)$ there is an $O(m(N) \sqrt{N} / f(N))$ combinatorial algorithm for the second shortest simple path in directed unweighted graphs. Then, let $L$ be such that $m(n+$ 
$4 L(L+1))=O\left(n^{2}\right)$. One can find a triangle using a combinatorial algorithm in time

$$
O\left(n / L \cdot\left(n^{2} L\right) / f\left(L^{2}\right)\right)=O\left(n^{3} / f\left(L^{2}\right)\right) \leq O\left(n^{3} / f(n)\right) .
$$

If $f(n)$ is a polynomial, then there is a truly subcubic combinatorial algorithm for BMM.

\section{G.3 Two New BMM Algorithms}

Our results allow us to provide two new algorithms for BMM, relying on the relationship between BMM and triangle detection.

\section{G.3.1 Output-Sensitive Quantum BMM}

In the theory of quantum computing there are many fantastic results. One of these is that a triangle in a graph on $n$ nodes can be found using only $\tilde{O}\left(n^{1.3}\right)$ operations [MSS05]. Recently, Buhrman and Spalek [Bv06] studied the problem of verifying and computing matrix products using a quantum algorithm. Among other nice results, their paper showed an $\tilde{O}\left(n^{1.5} \sqrt{L}\right)$ output-sensitive algorithm for computing the Boolean matrix product of two $n \times n$ matrices, where $L$ is the number of ones in the output matrix. Lemma 3.2 is a black box reduction which implies an improved algorithm by plugging in Magniez, Santha and Szegedy's [MSS05] triangle algorithm.

Lemma G.1 There is an $\tilde{O}\left(n^{1.3} L^{17 / 30}\right)$ quantum algorithm for computing the Boolean matrix product of two $n \times n$ matrices, where $L$ is the number of ones in the output matrix.

Notice that since $L \leq n^{2}$, we always have $n^{1.3} L^{17 / 30}<<\tilde{O}\left(n^{1.5} \sqrt{L}\right)$.

Proof of Lemma G.1. Let $A$ and $B$ be the given Boolean matrices. Consider a tripartite graph with partitions $I, J, K$ so that for $i \in I, j \in J(i, j)$ is an edge iff $A[i, j]=1$, for $j \in J, k \in K,(j, k)$ is an edge iff $B[j, k]=1$ and $(i, k)$ is an edge for all $i \in I, k \in K$. The graph does not need to be created explicitly whenever the algorithm has a query whether $(a, b)$ is an edge in the graph, it can just query $A$ and $B$, and any output it has already produced. Then, in the output-sensitive part of the proof of Lemma 3.2, we can just use $T(n)=\tilde{O}\left(n^{1.3}\right)$ given by the algorithm of [MSS05]. Notice that the condition of the lemma is satisfied for $T(n)=\tilde{O}\left(n^{1.3}\right)$. Hence we get an algorithm with quantum complexity $\tilde{O}\left(n^{1.3} L^{1-1.3 / 3}\right)=\tilde{O}\left(n^{1.3} L^{17 / 30}\right)$.

Using the improved output-sensitive algorithm from Theorem G.3 the above runtime can be modified to be $\tilde{O}\left(n^{2}+L^{47 / 60} n^{13 / 15}\right)$ which is better than the result above for all $L \geq \Omega\left(n^{1.24}\right)$. We prove Theorem 1.5 .

Reminder of Theorem 1.5 There is an $\tilde{O}\left(\min \left\{n^{1.3} L^{17 / 30}, n^{2}+L^{47 / 60} n^{13 / 15}\right\}\right)$ quantum algorithm for computing the Boolean matrix product of two $n \times n$ matrices, where $L$ is the number of ones in the output matrix.

\section{G.4 Polynomial Preprocessing and Faster Combinatorial BMM}

The divide-and-conquer ideas in our theorems are admittedly quite simple, but they are also powerful. It is evident that these ideas are useful for solving function problems via algorithms for related decision problems. These ideas can also be applied to greatly relax the conditions needed to achieve faster algorithms for the decision problems themselves. Williams [Wil07] showed that it is possible to preprocess a graph in $O\left(n^{2+\varepsilon}\right)$ time (for all $\varepsilon>0$ ) such that queries of the form is $S$ an independent set? can be answered in $O\left(n^{2} / \log ^{2} n\right)$ time. This data structure can be easily used to solve triangle detection in $O\left(n^{3} / \log ^{2} n\right)$, 
by simply querying the neighborhoods of each vertex. Bansal and Williams [BW09] show that every graph can be (randomly) preprocessed in $O\left(n^{2+\varepsilon}\right)$ time so that any batch of $O(\log n)$ independent set queries can be answered in $O\left(n^{2} / \log ^{1.25} n\right)$ (deterministic) time. This implies an $O\left(n^{3} / \log ^{2.25} n\right)$ randomized triangle detection algorithm. A major limitation in this approach to fast triangle detection is that the preprocessing time apparently must be subcubic. In fact, this subcubic requirement is the only reason why Bansal and Williams' preprocessing algorithm needs randomization. It turns out that in fact any polynomial amount of preprocessing suffices:

Reminder of Theorem 4.2 Suppose there are $k, c>0$ such that every n-node graph can be preprocessed in $O\left(n^{k}\right)$ time so that all subsequent batches of $O(\log n)$ independent set queries $S_{1}, \ldots, S_{\log n}$ can be answered in $O\left(n^{2} / \log ^{c} n\right)$ time. Then triangle detection (and hence Boolean matrix multiplication) is solvable in $O\left(n^{3} / \log ^{c+1} n\right)$ time.

That is, in order to attain better combinatorial algorithms for BMM, it suffices to answer independent set queries quickly with any polynomial amount of preprocessing. Theorem 4.2 holds for both randomized and deterministic algorithms: a deterministic preprocessing and query algorithm results in a deterministic BMM algorithm.

Proof of Theorem 4.2. Let $a=1 /(2 k)$. Divide the $n$ nodes of the graph into $n^{1-a}$ parts, each part having at most $2 n^{a}$ nodes each. For each pair $i, j$ of parts, let $G_{i, j}=\left(V_{i, j}, E_{i, j}\right)$ be the subgraph of $G$ restricted to the nodes in parts $i$ and $j$. Preprocess $G_{i, j}$ for independent set queries in $O\left(n^{a k}\right)$ time. This stage takes $O\left(n^{2(1-a)+a k}\right) \leq n^{2-1 / k+1 / 2} \leq O\left(n^{2.5}\right)$ time.

To determine if $G$ has a triangle, partition the set of nodes of $G$ into $n / \log n$ groups of $O(\log n)$ nodes earch. For each group $v_{1}, \ldots, v_{\log n}$ and all pairs of indices $i, j=1, \ldots, n^{1-a}$, and query $N\left(v_{1}\right) \cap$ $V_{i, j}, \ldots, N\left(v_{\log n}\right) \cap V_{i, j}$ for independence. If any query answers "no" then report that there is a triangle;if all queries answer "yes" over all nodes then report that there is no triangle. This stage takes $O\left(n / \log n \cdot n^{2(1-a)}\right.$. $\left.n^{2 a} /\left(a \log ^{c} n\right)\right) \leq O\left(n^{3} / \log ^{c+1} n\right)$ time.

Theorem 4.2 makes it easy to give derandomized versions of Bansal and Williams' algorithms, since there are deterministic polynomial time algorithms for the problems they need to solve, just not subcubic ones.

Reminder of Theorem 1.4 There is a deterministic combinatorial algorithm for BMM running in $O\left(n^{3} / \log ^{2.25} n\right)$ time.

Proof of Theorem 1.4. We will show that there is a deterministic combinatorial $O\left(n^{3} / \log ^{2.25} n\right)$ time algorithm for triangle finding. By Corollary 3.1 this also gives a deterministic combinatorial $O\left(n^{3} / \log ^{2.25} n\right)$ time algorithm for BMM.

The preprocessing algorithm of Bansal and Williams (Theorem 5.1 in [BW09]) proceeds by finding an $\varepsilon$ pseudoregular partition in the sense of [FK99] in $O\left(n^{2}\right)$ randomized time. The resulting independent set query algorithm answers $O(\log n)$ independent set queries in $O\left(n^{2} / \log ^{1.25} n\right)$ time and is completely deterministic. Alon and Naor [AN06] give a deterministic polynomial time algorithm for computing an $\varepsilon$-pseudoregular partition, which works for all $\varepsilon \leq c / \sqrt{\log n}$ for a fixed constant $c>0$. By replacing the randomized preprocessing with the algorithm of Alon and Naor and applying Theorem 4.2, we obtain the result.

Using the connection between negative triangle and APSP, we can identify a natural query problem on weighted graphs whose solution would give faster APSP algorithms. On a graph with an edge weight function $c: E \rightarrow \mathbb{Z}$, define a price query to be an assignment of node weights $p: V \rightarrow \mathbb{Z}$, where the answer to a query is yes if and only if there is an edge $(u, v) \in E$ such that $p(u)+p(v)>c(u, v)$. Intuitively, think of $p(v)$ as 
a price on node $v$, the edge weight $c(u, v)$ as the cost of producing both $u$ and $v$, and we wish to find for a given list of prices if there is any edge we are willing to "sell" at those prices.

Reminder of Theorem 4.3 Suppose there are $k, c>0$ such that every n-node edge-weighted graph can be preprocessed in $O\left(n^{k}\right)$ time so that any price query can be answered in $O\left(n^{2} / \log ^{c} n\right)$ time. Then negative triangle detection is solvable in $O\left(n^{3} / \log ^{c} n\right)$ time (and hence APSP is solvable in $O\left(n^{3} \log W / \log ^{c} n\right)$ time.

To some, the contrapositive of Theorem 4.3 may be more interesting: assuming that APSP needs $\Omega\left(n^{3} / \operatorname{poly} \log n\right)$ time, there is a super-polynomial lower bound on the preprocessing time for efficiently answering price queries.

\section{H A Simplified View of All-Pairs Path Problems and Their Matrix Products}

In this section we consider various algebraic structures other than the $(\mathrm{min},+)$ and Boolean semirings. We relate their matrix products and respective triangle problems, showing how several prior results in the area can be simplified in a uniform way.

Existence-Dominance. The dominance product of two integer matrices $A$ and $B$ is the integer matrix $C$ such that $C[i, j]$ is the number of indices $k$ such that $A[i, k] \leq B[k, j]$. The dominance product was first studied by Matoušek [Mat91] who showed that for $n \times n$ matrices it is computable in $O\left(n^{(3+\omega) / 2}\right)$. The existence-dominance product of two integer matrices $A$ and $B$ is the Boolean matrix $C$ such that $C[i, j]=0$ iff there exists a $k$ such that $A[i, k] \leq B[k, j]$. This product was used in the design of the first truly subcubic algorithm for the minimum node-weighted triangle problem [VW06]. Although the existence-dominance product seems easier than the dominance product, the best known algorithm for it actually computes the dominance product.

The existence-dominance product is defined over the (min, $\odot$ ) structure for which $R=\mathbb{Z} \cup\{-\infty, \infty\}$ and $a \odot b=0$ if $a \leq b$ and $a \odot b=1$ otherwise. The corresponding negative triangle problem, the dominance triangle problem, is defined on a tripartite graph with parts $I, J, K$. The edges between $I$ and $J$ are unweighted, and the rest of the edges in the graph have real weights. The goal is to find a triangle $i, j, k \in I \times J \times K$ such that $w(i, k) \leq w(k, j)$.

Minimum Edge Witness. The minimum edge witness product is defined over a restriction of the $(\mathrm{min}, \odot)$ structure over $R=\mathbb{Z} \cup\{\infty,-\infty\}$, where $\odot=\times$ is integer multiplication. For an integer matrix $A$ and a $\{0,1\}$ matrix $B$, the $(i, j)$ entry of the minimum edge witness product $C$ of $A$ and $B$ is equal to $\min _{k}(A[i, k] \times$ $B[k, j])$. This product is important as it is in truly subcubic time iff APSP on node-weighted graphs is in truly subcubic time. Chan [Cha07] used this relation to obtain the first truly subcubic runtime for node-weighted APSP.

The negative triangle problem corresponding to the minimum edge witness product is again the dominance triangle problem. Hence, by Theorem 3.2 we can conclude that a truly subcubic algorithm for the dominance triangle problem (such as Matoušek's algorithm for the dominance product) implies truly subcubic node-weighted APSP. That is, we get an alternative subcubic algorithm for node-weighted APSP as a byproduct, although it is a bit slower than the best known. To obtain his algorithm for node-weighted APSP, Chan [Cha07] gave a completely new algorithm for minimum edge witness product with exactly the same runtime as Matoušek's dominance product algorithm. 
(Min- $\leq$ ). The $(\min , \leq)$ structure is defined over $R=\mathbb{Z} \cup\{\infty,-\infty\}$, where the binary operation $\leq$ on input $a, b$ returns $b$ if $a \leq b$ and $\infty$ otherwise. The first author showed [Vas08] that the (min, $\leq)$ matrix product is in truly subcubic time iff the all pairs minimum nondecreasing paths problem (also called earliest arrivals) is in truly subcubic time. The first truly subcubic runtime for the product, $O\left(n^{2+\omega / 3}\right)$, was obtained by the present authors and R. Yuster [VWY07]. The techniques of Duan and Pettie [DP09] also imply an $O\left(n^{(3+\omega) / 2}\right)$ algorithm.

The negative triangle problem over $(\min , \leq)$ is the following nondecreasing triangle problem: given a tripartite graph with partitions $I, J, K$ and real edge weights, find a triangle $i \in I, j \in J, k \in K$ such that $w(i, k) \leq w(k, j) \leq w(i, j)$.

Both known algorithms for this problem follow from the algorithms for (min, $\leq$ )-product [VWY07, DP09] and are somewhat involved. Below we give a simpler $O\left(n^{3 / 2} \sqrt{T(n)}\right)$ algorithm, where $T(n)$ is the best runtime for finding a triangle in an unweighted graph. If matrix multiplication is used, the runtime is the same as in Duan-Pettie's algorithm, $O\left(n^{(3+\omega) / 2}\right)$. Furthermore, the algorithm can actually be applied $O(\log n)$ times to obtain another $\tilde{O}\left(n^{(3+\omega) / 2}\right)$ algorithm for the $(\mathrm{min}, \leq)$-product.

Theorem H.1 (Nondecreasing Triangle $\leq_{3}$ Triangle) If a triangle in an unweighted graph can be found in $T(n)$ time, then a nondecreasing triangle can be found in $O\left(n^{3 / 2} \sqrt{T(O(n))}\right)$ time, and $(\mathrm{min}, \leq)$ product is in $O\left(n^{3 / 2} \sqrt{T(O(n))} \log n\right)$ time.

Proof. We are given a weighted tripartite graph with partitions $I, J, K$ and are looking for a triangle $i \in I, j \in J, k \in K$ such that $w(i, k) \leq w(k, j) \leq w(i, j)$.

Begin by sorting all the edges in the graph, breaking ties in the following way: edges from $I \times J$ are considered bigger than edges from $K \times J$ of the same weight which are considered bigger than edges from $I \times K$ of the same weight; within $I \times J$ or $J \times K$ or $I \times K$ equal edges are arranged arbitrarily.

Let $t$ be a parameter. For every vertex $v$ in $J$ or $K$, consider the sorted order of edges incident to $v$ and partition it into at most $n / t$ buckets of $t$ consecutive edges each and at most one bucket with $\leq t$; let $B_{v b}$ denote the $b$-th bucket for node $v$. For each edge $(x, v)$ such that $v$ is in $J$ or $K$ and $(x, v)$ is in $B_{v b}$, go through all edges $(v, y)$ in $B_{v b}$ and check whether $x, v, y$ forms a nondecreasing triangle. This takes $O\left(n^{2} t\right)$ time.

Partition the edges of the graph by taking $O(n / t)$ consecutive groups of $\leq n t$ edges in the sorted order of all edges. Let $G_{g}$ denote the $g$-th such group. For each $g$, consider all buckets $B_{v b}$ of vertices $v$ in $J$ or $K$ such that there is some edge $(v, x) \in B_{v b} \cap G_{g}$. There can be at most $4 n$ such buckets: there are at most $n+n t / t=2 n$ buckets completely contained in $G_{g}$ and at most $2 n$ straddling $G_{g}-$ at most one per vertex per group boundary.

Create a tripartite graph $H_{g}$ for each $g$ as follows. $H_{g}$ has partitions $H_{g}^{I}, H_{g}^{J}$ and $H_{g}^{K} . H_{g}^{I}$ has a node for each $i \in I$. For $S \in\{J, K\} H_{g}^{S}$ has a node for each node bucket $B_{v b}$ such that $B_{v b} \cap G_{g} \neq \emptyset$ and $v \in S$. Therefore $H_{g}$ has $\leq 9 n$ nodes.

The edges of $H_{g}$ are as follows. For all $B_{j b} \in H_{g}^{J}$ and $B_{k b^{\prime}} \in H_{g}^{K},\left(B_{j b}, B_{k b^{\prime}}\right)$ is an edge if $(j, k)$ is an edge and it is in $B_{j b} \cap B_{k b^{\prime}}$. For $i \in H_{g}^{I}$ and $B_{j b} \in H_{g}^{J},\left(i, B_{j b}\right)$ is an edge in $H_{g}$ iff $(i, j) \in E$ and there is a bucket $b^{\prime}<b$ such that $(i, j) \in B_{j b^{\prime}}$. For $i \in H_{g}^{I}$ and $B_{k b} \in H_{g}^{K},\left(i, B_{k b}\right)$ is an edge in $H_{g}$ iff $(i, k) \in E$ and there is a bucket $b^{\prime}>b$ such that $(i, k) \in B_{k b^{\prime}}$.

Any triangle $i, B_{j b}, B_{k, b^{\prime}}$ in $H_{g}$ corresponds to a nondecreasing triangle $i, j, k$ in $G$. If a nondecreasing triangle $i, j, k$ of $G$ is not contained in any $H_{g}$, then for some $b$ either both $(i, j)$ and $(j, k)$ are in $B_{j b}$ or both $(i, k)$ and $(j, k)$ are in $B_{k b}$, both cases of which are already handled. 
The runtime is $O\left(n^{2} t+T(9 n) \cdot n / t\right)$. Setting $t=\sqrt{T(9 n) / n}$, the time becomes $O\left(n^{3 / 2} \sqrt{T(9 n)}\right)$.

Min-Max. The subtropical semiring $(\min , \max )$ is defined over $R=\mathbb{Z} \cup\{\infty,-\infty\}$. The (min, $\max$ ) matrix product was used by the present authors and R. Yuster [VWY07] to show that the all pairs bottleneck paths problem is in truly subcubic time. The current best algorithm for the problem runs in $O\left(n^{(3+\omega) / 2}\right)$ time by Duan and Pettie [DP09]. The (min, max) product is an important operation in fuzzy logic, where it is known as the composition of relations ([DP80], pp.73).

The negative triangle problem over $(\min , \max )$ is the following $I J$-bounded triangle problem. Given a tripartite graph with partitions $I, J, K$ and real weights on the edges, find a triangle $i \in I, j \in J, k \in K$ such that both $w(i, k) \leq w(i, j)$ and $w(j, k) \leq w(i, j)$, i.e. the largest triangle edge is in $I \times J$. We note that any algorithm for the nondecreasing triangle problem also solves the $I J$-bounded triangle problem: any $I J$-bounded triangle appears as a nondecreasing triangle either in the given graph, or in the graph with partitions $J$ and $K$ swapped. Hence a corollary to Theorem $\mathrm{H}$.1 is that an $I J$-bounded triangle can be found in $O\left(n^{3 / 2} \sqrt{T(n)}\right)$ time, where $T(n)$ is the runtime of a triangle detection algorithm for unweighted graphs.

\section{Extension to 3SUM}

Finally, we describe an application of the ideas in this paper to the 3SUM problem. We show that the 3SUM problem has a truly subquadratic algorithm if and only if the All-Ints-3SUM problem does. In both problems, one is given a list $A$ of $n$ integers each. In All-Numbers-3SUM one needs to return all integers $c$ in $A$ such that there are $a, b \in A$ with $a+b+c=0$, and for 3SUM one merely has to detect whether one such $c$ exists.

The All-Ints 3SUM problem is a generalization of the 3SUM problem: given three lists $A, B, C$ of $n$ integers each, one wants to determine all integers $a \in A$ such that there exist $b \in B, c \in C$ with $a+b+c=0$. We show that our techniques can be used to show the theorem below.

Theorem I.1 (All-Ints 3SUM $\equiv_{2}$ 3SUM) All-Ints $3 S U M$ is in truly subquadratic time iff $3 S U M$ is in truly subquadratic time.

Proof. We first use a hashing scheme given by Dietzfelbinger [Die96] and used by Baran, Demaine and Patrascu [BDP08] which maps each distinct integer independently to one of $\sqrt{n}$ buckets 8 . For each $i \in[\sqrt{n}]$, let $A_{i}, B_{i}$, and $C_{i}$ be the sets containing the elements hashed to bucket $i$. The hashing scheme has two nice properties:

1. for every pair of buckets $A_{i}$ and $B_{j}$ there are two buckets $C_{k_{i j 0}}$ and $C_{k_{i j 1}}$ (which can be located in $O(1)$ time given $i, j$ ) such that if $a \in A_{i}$ and $b \in B_{j}$, then if $a+b \in C$ then $a+b$ is in either $C_{k_{i j 0}}$ or $C_{k_{i j 1}}$,

2. the number of elements which are mapped to buckets with at least $3 \sqrt{n}$ elements is $O(\sqrt{n})$ in expectation.

After the hashing we process all elements that get mapped to large buckets (size $>3 \sqrt{n}$ ). Suppose $a \in A$ is such an element (WLOG it is in $A$ ). Then go through all elements $b$ of $B$ and check whether $a+b \in C$. This takes $O\left(n^{1.5}\right)$ time overall in expectation.

\footnotetext{
${ }^{8}$ The scheme performs multiplications with a random number and some bit shifts hence we require that these operations are not too costly. We can ensure this by first mapping the numbers down to $O(\log n)$ bits, e.g. by computing modulo some sufficiently large $\Theta(\log n)$ bit prime.
} 
Now the buckets $A_{i}, B_{i}, C_{i}$ for all $i \in[\sqrt{n}]$ contain $O(\sqrt{n})$ elements each. In particular, we have reduced the problem to $2 n$ subinstances of $3 \operatorname{SUM}\left(\left(A_{i}, B_{j}, C_{k_{i j b}}\right)\right.$ for $\left.b=0,1\right)$. For every one of these $2 n$ subinstances, in turn: Call the detection algorithm. We can assume that the detection algorithm actually returns a triple $a \in A_{i}, b \in B_{j}, c \in C_{k_{i j b}}$ which is a 3SUM (by a self-reduction, halving argument). Remove $a$ from $A_{i}$, recording that it is in a 3SUM. Try to find a new 3SUM in the subinstance. Move on to the next subinstance if the current one does not contain any more solutions.

Assuming that there is an $O\left(n^{2-\varepsilon}\right)$ 3SUM detection algorithm, the running time from this portion of the reduction becomes asymptotically

$$
(n+2 n) \cdot(\sqrt{n})^{2-\varepsilon}=O\left(n^{2-\varepsilon / 2}\right),
$$

and so All-Ints-3SUM can be solved in $O\left(n^{1.5}+n^{2-\varepsilon / 2}\right)$ time. 